\title{
Dual pH-sensitive liposomes with low pH-triggered sheddable PEG for enhanced tumor-targeted drug delivery
}

\author{
Manju Kanamala1, Brian D Palmer², Stephen MF Jamieson², William R Wilson² \& Zimei \\ Wu*,1 \\ ${ }^{1}$ School of Pharmacy, Auckland Cancer Society Research Centre, Faculty of Medical \& Health Sciences, University of Auckland, \\ Auckland 1142, New Zealand \\ ${ }^{2}$ Auckland Cancer Society Research Centre, Faculty of Medical \& Health Sciences, University of Auckland, Auckland 1142, New \\ Zealand \\ *Author for correspondence: Tel.: + 649 9231709; z.wu@auckland.ac.nz
}

Aim: $\mathrm{pH}$-sensitive liposomes ( $\mathrm{pSL}$ ) have emerged as promising nanocarriers due to their endo/lysosomeescape abilities, however, their pH sensitivity is compromised by poly(ethylene glycol) (PEG) coating. This study investigates whether an intracellular PEG-detachment strategy can overcome this PEG dilemma. Materials \& methods: First, PEG2000 was conjugated with a phospholipid via an acid-labile hydrazidehydrazone bond (-CO-NH-N = CH-), which was postinserted into pSL, forming PEG-cleavable pSL (CL-PEGpSL). Their endo/lysosomal-escape abilities in MIA PaCa-2 cells, pharmacokinetics and tumor accumulation abilities were studied using PEG-pSL as reference. Results: CL-PEG-pSL showed rapid endo/lysosomeescape abilities in the cancer cells and higher tumor accumulation in MIA PaCa-2 xenograft model in contrast to PEG-pSL. Conclusion: Cleavable PEGylation is an efficient strategy to ameliorate the PEG dilemma of $\mathrm{pSL}$ for cancer drug delivery.

\section{Graphical abstract:}

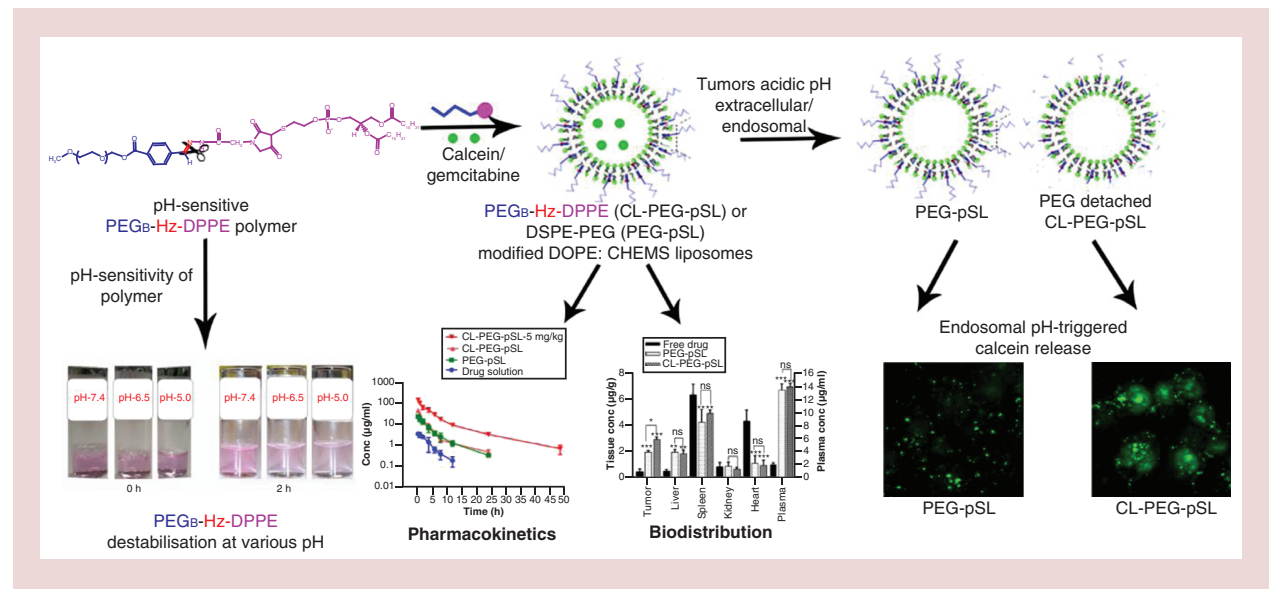

First draft submitted: 21 December 2018; Accepted for publication: 8 April 2019; Published online: 29 July 2019

Keywords: dual pH-sensitive liposomes • endosome escape • live cell imaging • MIA PaCa-2 • PEG-detachment • PEG dilemma • PEG shedding • pharmacokinetics • tumor distribution

Phosphatidylethanolamine (PE)-based pH-sensitive liposomes (pSL) have been widely investigated for efficient, tumor-targeted drug and gene delivery [1-5], due to their fusogenic properties and abilities to exploit enhanced permeation and retention effect [6,7]. Along with the poorly hydrated PE head group, these pSL can be converted to an inverted hexagonal phase on exposure to acidic $\mathrm{pH}$, thus forming destabilized nonlamellar struc- 
tures, resulting in content release $[3,8,9]$. The most commonly used lipid combination is 1,2-dioleoyl-sn-glycero3-phosphoethanolamine (DOPE) with cholesteryl hemisuccinate (CHEMS) [10,11]. Due to their fusogenic and $\mathrm{pH}$-sensitive properties, DOPE-CHEMS liposomes exploit the tumor extracellular $\mathrm{pH}\left(\mathrm{pH}_{\mathrm{ex}} ; 6.4-7.4\right.$ [5]) for preferential cellular uptake, and mostly, the endo/lysosomal $\mathrm{pH}\left(\mathrm{pH}_{\mathrm{en}} ; 5.0-6.5\right)$ to undergo rapid endo/lysosomalescape, resulting in preferential release of the payloads at the target in cells [12]. Surface coating with PEG, in other words, PEGylation, can prolong the circulation half-life of liposomes by preventing their rapid clearance by the reticuloendothelial system or their destabilization through interactions with serum proteins [13]. The primary emphasis so far has been on the development of PEG-coated pSL, where the PEG layer promotes long circulation by preventing opsonization and $\mathrm{pH}$-sensitive lipids facilitate release of the contents in the acidic extracellular space and also through more efficient endosomal escape within the cancer cells $[2,5,14,15]$.

However, growing evidence shows that hydrophilic PEG coating of liposomes hinders drug release [16], reduces interaction with target cells [17] and, in case of pSL, limits the endosomal escape property [18,19], and can therefore compromise in the therapeutic response of the loaded drug. This is known as the 'PEG dilemma' $[18,20]$. A common approach, as for conventional liposomes, to achieving tumor-selective cellular uptake is by coating the surfaces of pSL with targeting ligands specific to cancer cells [21]. However, these surface functionalization strategies require gentler chemistries, which are mostly not stable in the complex in vivo environment [22].

Alternatively, attempts have been made to design tumor microenvironment-triggered cleavable PEGs, which have the potential to exploit the low $\mathrm{pH}_{\mathrm{ex}}$ of tumors [23-25]. However, various challenges have been reported to date. Torchilin et al. investigated liposomes coated with aliphatic and aromatic aldehyde-derived hydrazone ( $\mathrm{Hz})$-based acid-sensitive cleavable PEG-1,2-dipalmitoyl-sn-glycero-3-phosphoethanolamine (DPPE) polymers, and found that the aliphatic aldehyde-based PEG conjugates, although destabilized at pH 5.5 with a short half-life of 2 min were, unfortunately, also unstable at physiologic $\mathrm{pH}$ (half-life $2.5 \mathrm{~h}$ ). In contrast, those derived from aromatic aldehydes were stable enough at $\mathrm{pH} 7.4$ (half-life of $72 \mathrm{~h}$ ) but less $\mathrm{pH}$-sensitive with a half-life of $48 \mathrm{~h}$ at $\mathrm{pH} 5.5$ [24]. More recently, Chen et al. reported on the design of PEG detachable liposomes (non-pH-sensitive bilayers) using mPEG-Hz-CHEMS based on ketone-derived hydrazone to achieve enhanced cellular uptake and cytotoxicity [25]. However, these novel liposomes showed a higher accumulation in liver and spleen compared with the conventional liposomes due to the poor stability of the polymer at physiological $\mathrm{pH}$. Our previous research showed that $\mathrm{PEG}_{\mathrm{B}}$ $\mathrm{Hz}$-CHEMS-modified liposomes do have satisfactory stability and $\mathrm{pH}$-responsive PEG-detachment; however, they have poor insertion abilities into liposomes, which compromise the stealth abilities of the resulting liposomes [26]. This could be due to the small cholesterol-like hydrophobic heads, which favor formation of micelles. Therefore, we inferred that PEG anchored on the head groups of phospholipids may overcome the above challenges.

In this paper, we designed a novel acid-labile $\mathrm{PEG}_{\mathrm{B}}-\mathrm{Hz}-\mathrm{DPPE}$ conjugate via an acid-labile hydrazide-hydrazone $(-\mathrm{CO}-\mathrm{NH}-\mathrm{N}=\mathrm{CH}-)$ bond. We further demonstrated a dual $\mathrm{pH}$-responsive strategy, where $\mathrm{PEG}_{\mathrm{B}}-\mathrm{Hz}-\mathrm{DPPE}$ was coated on a pSL, forming cleavable PEG-coated pSL (CL-PEG-pSL). This system takes advantage of both the acid-labile sheddable PEG layer and the $\mathrm{pH}$-sensitive lipid bilayer that promotes the rapid intracellular trafficking. The dual pSL strategy with PEG $_{B}-\mathrm{Hz}$-CHEMS has been investigated previously [26], and little is known about their endosome escape kinetics of PEG cleavable pSL. Thus, in this study, live cell imaging technique was employed to reveal the detailed intracellular trafficking of CL-PEG-pSL. The influence of cleavable PEG on the in vitro/in vivo performance of CL-PEG-pSL, including drug release, intracellular accumulation, endosomal escape, cytotoxicity to MIA PaCa-2 pancreatic cancer and U-87 MG glioblastoma cells, pharmacokinetics and tissue distribution, were subsequently investigated with gemcitabine as a model drug in comparison to conventional PEGylated pSL (PEG-pSL) with the same degree of PEGylation.

\section{Materials \& methods \\ Materials}

Methoxy PEG (mPEG, $\left.M_{n}=2000\right)$, cholesterol, Sephadex G-25, calcein and Nile Red were acquired from Sigma-Aldrich (MO, USA). Phospholipids such as DOPE, 1,2-distearoyl-sn-glycero-3-phosphocholine (DSPC), DPPE, 1,2-dipalmitoyl-sn-glycero-3-phosphoethanolamine- $N$-(lissamine rhodamine B sulfonyl; ammonium salt; Rh-PE) and CHEMS were purchased from Avanti Polar Lipids (AL, USA). Dicyclohexylcarbodiimide, 4-(dimethylamino)pyridine and $\mathrm{N}-\varepsilon$-maleimidocaproic acid hydrazide $(\mathrm{EMCH})$ were purchased from Thermo Fisher Scientific Inc. (Auckland, New Zealand). Gemcitabine hydrochloride (99.95\% purity) was obtained from Selleckchem (TX, USA). Milli-Q water (HPLC grade) was obtained from a Millipak filter unit (Millipore, pore size: $0.22 \mu \mathrm{m}$; Auckland, New Zealand). 
The MIA PaCa-2 pancreatic cancer cells and glioblastoma U-87 MG cells were obtained from the American Type Culture Collection (ATCC, MD, USA). Gibco ${ }^{T M}$ DMEM cell culture medium, fetal bovine serum, penicillinstreptomycin-glutamine $(100 \times)$ were purchased from Thermo Fisher Scientific Inc. (Auckland, New Zealand).

\section{Synthesis of $\mathrm{PEG}_{\mathrm{B}}-\mathrm{Hz}-\mathrm{DPPE}$ polymer}

The synthesis of $\mathrm{PEG}_{\mathrm{B}}-\mathrm{Hz}$-DPPE was carried out with a three-step procedure. First, hydrazide-activated DPPE was synthesized according to our previous method with some modifications [24]. For step 1, DPPE (22 $\mu$ M) was mixed with 1.5 molar excess of EMCH hydrazide linker in $3 \mathrm{ml}$ of anhydrous methanol. Triethylamine (five mole excess over lipid) was added to the resulting reaction mixture to achieve $\mathrm{pH}$ of $6.5-7.5$, which activates the maleimide moiety of EMCH and the reaction mixture was stirred at $25^{\circ} \mathrm{C}$ overnight. The resulting reaction mixture was purified on silica gel column by eluting with chloroform:methanol $(3: 1 \mathrm{v} / \mathrm{v})$. The phosphate-containing turbid fractions were pooled, concentrated under reduced pressure, and the structure was confirmed by ${ }^{1} \mathrm{H}$ NMR spectroscopy (yield: 80\%). For step 2, $\mathrm{PEG}_{\mathrm{B}}$ was synthesized according to our previous method [26]. For step 3, $\mathrm{PEG}_{\mathrm{B}}(0.06 \mathrm{mmol})$ was mixed with DPPE-hydrazide $(0.09 \mathrm{mmol})$ in $2 \mathrm{ml}$ of chloroform at $25^{\circ} \mathrm{C}$. After overnight stirring, the resulting reaction mixture was evaporated to dryness and the obtained residue was purified by passing through a Sephadex G-25 column using purified water, $\mathrm{pH}$ 7.4. The fractions containing the compound were pooled, freeze dried and confirmed by ${ }^{1} \mathrm{H}$ NMR spectroscopy (yield: $84.1 \%$ ).

\section{Characterization of $\mathrm{PEG}_{\mathrm{B}}-\mathrm{Hz}-\mathrm{DPPE}$ polymer}

To determine the $\mathrm{pH}$-dependent bond cleavage properties of the polymer, the shift in the particle size of the polymeric micelles was monitored following their incubation at various $\mathrm{pH}$ values at $37^{\circ} \mathrm{C}$, using dynamic light scattering [27]. Briefly, the polymer (at a concentration of $2.0 \mathrm{mg} / \mathrm{ml}$ ) was incubated in various phosphate buffers $(0.01 \mathrm{M}, \mathrm{pH} 7.4,6.5$ and 5.0$)$ at $37^{\circ} \mathrm{C}$, with shaking at $120 \mathrm{rpm}$ for $2 \mathrm{~h}$. The size of the polymeric micelles was monitored using dynamic light scattering. Furthermore, the physical stability of micelles in phosphate-buffered saline (PBS; 0.01 M, pH 7.4) was determined by measuring their size and morphology using Malvern Nano Zeta Sizer (Malvern Instruments, UK) and Tecnai cryo-transmission electron microscopy (cryo-TEM; FEI, OR, USA).

The $\mathrm{pH}$-responsive destabilization behavior of $\mathrm{PEG}_{\mathrm{B}}-\mathrm{Hz}-\mathrm{DPPE}$ micelles was confirmed by a Nile Red assay [28]. To prepare Nile Red (a fluorescent probe)-loaded micelles, $30 \mathrm{mg}$ PEG $_{B}-\mathrm{Hz}-\mathrm{DPPE}$ and $0.2 \mathrm{mg}$ Nile Red were first dissolved in $2 \mathrm{ml}$ methanol, followed by addition of $10 \mathrm{ml}$ PBS, $0.01 \mathrm{M}$, pH 7.4 dropwise into the solution. After stirring for $8 \mathrm{~h}$ at $37^{\circ} \mathrm{C}$, excess of Nile Red was removed from the polymeric micelles by filtration through a $0.45-\mu \mathrm{m}$ membrane. $100 \mu \mathrm{l}$ of Nile Red-loaded micelle solution $\left(2 \mathrm{mg} \mathrm{m}^{-1}\right)$ was incubated in $900 \mathrm{ml}$ of $0.01 \mathrm{M}$ PBS, at pH 7.4, 6.5 and 5.0, respectively, and the change in color was monitored over time.

In addition, the Nile Red-incorporated micelle solution was used to determine the critical micellar concentration (CMC) of $\mathrm{PEG}_{\mathrm{B}}-\mathrm{Hz}-\mathrm{DPPE}$ by fluorescence spectroscopy [29]. Nile Red-incorporated polymeric micelles were diluted to a polymer concentration ranging from $2 \times 10^{-4}$ to $0.2 \mathrm{mM}$. Fluorescence measurement was carried out using a Fluoro Max-4 spectrophotometer (Horiba Scientific) at an excitation wavelength of $550 \mathrm{~nm}$ and the emission monitored from $636 \mathrm{~nm}$. The CMC of the polymer was calculated by plotting the fluorescence intensity against $\log$ polymer concentration.

\section{Preparation of the liposomes}

Both CL-PEG-pSL and control formulation PEG-pSL were prepared by the thin-film hydration-extrusion method followed by PEGylation via postinsertion as described previously [26]. PEG-pSL were prepared with DOPE, DSPC, CHEMS, cholesterol, at the molar ratio of 4:2:2:2 in addition to 5 mol\% of DSPE-PEG $2_{\mathrm{k}}$ to the total lipids. CL-PEG-pSL were prepared with the same composition of lipids but replacing DSPE-PEG ${ }_{2 k}$ with PEG $_{B}-\mathrm{Hz}_{-}$ DPPE. All lipids apart from the PEG polymers were dissolved in chloroform:methanol $(3: 1 \% \mathrm{~V} / \mathrm{V})$, and then solvent was removed on rotary evaporator at $30^{\circ} \mathrm{C}$ under vacuum to form thin lipid films, which was kept under vacuum overnight. The resulting lipid films were hydrated with PBS (0.01 M, pH 7.4, added with $\mathrm{NaCl}$ to achieve iso-osomotic environment inside liposomes) at $30^{\circ} \mathrm{C}$. The resulting suspension was subjected to seven cycles of freeze and thaw and ten cycles of extrusion using a 10-ml LIPEX ${ }^{\top M}$ Extruder (Northern Lipids Inc., Burnaby, Canada) via $100-\mathrm{nm}$ membrane with a pressure of approximately 500 psi and a temperature of $50^{\circ} \mathrm{C}$, close to the $\mathrm{T}_{\mathrm{c}}$ of DSPC $\left(55^{\circ} \mathrm{C}\right)$.

The liposome pellets obtained following ultracentrifugation of the suspension were then PEGylated using the postinsertion technique [20,26]. For this, $1 \mathrm{ml}$ of PEG $\mathrm{B}_{\mathrm{H}} \mathrm{Hz}-\mathrm{DPPE}$ (for CL-PEG-pSL) or DSPE-PEG (for PEG-pSL) 
micelle suspension (equivalent to $5 \mathrm{~mol} \%$ to lipids) in PBS (0.01 M, pH 7.4) was used to resuspend the liposome pellet, which was incubated at $37^{\circ} \mathrm{C}$ for $2 \mathrm{~h}$ with continuous shaking at $350 \mathrm{rpm}$ to form CL-PEG-pSL and PEG-pSL.

Gemcitabine was loaded into the selected CL-PEG-pSL and PEG-pSL using a previously reported small volume incubation method [30]. Briefly, the liposome pellet was incubated with $20 \mu \mathrm{l}$ of gemcitabine suspension $(1.5 \mathrm{mg} / \mathrm{ml}$ gemcitabine in phosphate buffer, $\mathrm{pH} 7.4$ ), at $60^{\circ} \mathrm{C}$ for $3 \mathrm{~h}$. Free drug was removed by eluting the liposomal suspension through a gel filtration Sephadex G-75 column $(100 \times 20 \mathrm{~mm})$ using $20 \mathrm{mM}$ PBS containing $0.15 \mathrm{M}$ $\mathrm{NaCl}$ at $\mathrm{pH} 7.4$.

Doxorubicin was loaded into liposomes using the ammonium sulfate gradient method [31]. For this, lipid films were hydrated with a $250-\mathrm{mM}$ ammonium sulfate solution with $\mathrm{pH}$ being adjusted to 7.2 at $30^{\circ} \mathrm{C}$. Free ammonium sulfate was removed by dialysis, and the liposomes were collected by ultracentrifugation. Finally, the liposome pellet was resuspended with $1 \mathrm{mg} / \mathrm{ml}$ of doxorubicin solution in PBS, $\mathrm{pH} 7.9$ and incubated for $1 \mathrm{~h}$ at $37^{\circ} \mathrm{C}$ and the nonencapsulated doxorubicin was removed by ultracentrifugation.

For cellular uptake and intracellular trafficking studies, liposomes were labeled with Nile Red in the bilayer and calcein in the cores. Nile red $(0.2 \mu \mathrm{g} / \mathrm{ml})$ or Rh-PE $(0.5 \mathrm{mg} / \mathrm{ml})$ was added during thin-film preparation, which was further hydrated with calcein solution $(80 \mathrm{mM}, \mathrm{pH}$ adjusted to 7.4$)$

\section{Characterization of liposomes}

To determine the insertion efficiency of the $\mathrm{PEG}_{\mathrm{B}}-\mathrm{Hz}$-DPPE polymer, Nile Red-loaded polymeric micelles were prepared as described in 'Characterization of PEGB-Hz-DPPE Polymer' and were incubated with the non-PEGylated liposomes for $2 \mathrm{~h}$. The resulting liposome suspension was then ultracentrifuged to spin down the liposome pellet and was visually monitored for the color of the supernatant. Polymer-inserted liposome pellet was dissolved in $\mathrm{CDCL}_{3}$ and analyzed by NMR spectroscopy for further confirmation of polymer insertion into liposomes.

Entrapment efficiency (EE) and drug loading (DL) of drug-loaded liposomes were determined using the following equations, respectively.

$$
\begin{aligned}
& E E(\%)=\frac{\text { mass of drug in liposomes }}{\text { mass of drug used for loading }} \times 100 \\
& D L(\%)=\frac{\text { mass of drug in liposomes }}{\text { mass of drug loaded liposomes }} \times 100
\end{aligned}
$$

The mean size and surface charge of the liposomes were determined using a Malvern Zetasizer (Malvern Instruments, Germany). Particle size and zeta potential for non-PEGylated and PEGylated liposomes were compared to confirm the surface modification of liposomes with PEG. The morphology of the CL-PEG-pSL was observed by cryo-TEM.

\section{$\mathrm{pH}$-responsiveness of gemcitabine release}

The $\mathrm{pH}$-responsive drug release of gemcitabine-loaded PEG-pSL and CL-PEG-pSL was determined by the dialysis method. The drug-loaded liposomes were dispersed in $1 \mathrm{ml}$ of PBS $(50 \mathrm{mM}, \mathrm{pH} 7.4)$ and tightly sealed in cellulose acetate dialysis tubes (MW 12-14 kDa). Then, the dialysis tubes were immersed in $50 \mathrm{ml}$ of release medium (PBS $50 \mathrm{mM}, \mathrm{pH} 7.4$, adjusted with $\mathrm{NaCl}$ to achieve iso-osmotic environment, $320 \mathrm{mOsm}$ ), of different $\mathrm{pH} 7.4,6.5$ (representing $\mathrm{pH}_{\mathrm{ex}}$ ) and 5.0 (representing $\mathrm{pH}_{\mathrm{en}}$ ), and incubated under $37^{\circ} \mathrm{C}$ with shaking at $100 \mathrm{rpm}$ for $48 \mathrm{~h}$. Release media $(100 \mu \mathrm{l})$ were withdrawn and replaced with the same volume of fresh release media at various time intervals. The concentration of released gemcitabine was determined by HPLC [32].

The in vitro drug release-time profiles were compared using the similarity factor $\left(f_{2}\right)$ approach [33]. The $f_{2}$ value is a logarithmic transformation of the sum-squared error of the differences in \% release between two formulations $\left(T_{j}\right.$ and $R_{j}$ ) through all the time points. An $f_{2}$ value between 50 and 100 indicates the release profiles are similar and $f_{2}$ value of less than 50 indicate that profiles are not similar 
In vitro cellular uptake \& endosomal escape studies

For the qualitative study of the cellular uptake and endosome escape properties of CL-PEG-pSL and PEG-pSL, Nile Red and calcein dual-labeled liposomes were used. $1 \times 10^{5}$ cells per well were seeded in four-well chambered slides and cultured for $24 \mathrm{~h}$. The dual-labeled liposomes were added to the plates to a total lipid concentration of $0.4 \mathrm{mmol} / \mathrm{l}$ and incubated at $37^{\circ} \mathrm{C}$ for 1 and $2 \mathrm{~h}$. Cells were washed with cold PBS (pH 7.4) and fixed using $4 \%$ paraformaldehyde at $4^{\circ} \mathrm{C}$ for $10 \mathrm{~min}$. Finally, the nuclei were stained with DAPI for 5 min in the dark. Cells were observed under a confocal laser scanning microscope (CLSM, Olympus Fluroview FV1000, Olympus Corporation, Japan) with an excitation wavelength of $366 \mathrm{~nm}$ for DAPI, $488 \mathrm{~nm}$ for calcein and $546 \mathrm{~nm}$ for Nile Red. Fluorescence intensity of the images was further quantified using the ImageJ software.

\section{Subcellular localization of CL-PEG-pSL \& PEG-pSL with live cell imaging}

To further investigate the intracellular trafficking of the CL-PEG-pSL and PEG-pSL, live cell tracking studies were performed using the Olympus Fluroview FV1000 CLSM. MIA PaCa-2 cells were seeded at a density of $10^{3} /$ well in $300 \mu \mathrm{l}$ medium and allowed to attach overnight to ibidi 8-well chambered slides. The cells were stained by LysoTracker Deep Red $(75 \mu \mathrm{g} / \mathrm{ml})$ for $90 \mathrm{~min}$ and Hoechst $33342(1 \mathrm{mg} / \mathrm{ml})$ for $20 \mathrm{~min}$, both at $37^{\circ} \mathrm{C}$. A single cell with a clear morphology was chosen in a differential interference contrast (DIC) channel. Rh-PElabeled CL-PEG-pSL and PEG-pSL were then added to a total lipid concentration of $50 \mu \mathrm{g} / \mathrm{ml}$ and immediately observed using CLSM with a $60 \times$ oil immersion objective and live cell incubator system. Confocal images were acquired at regular intervals over a 2-h time period. Images were analyzed using ImageJ software, version 1.6 (MD, USA). The colocalization threshold plugin was used to calculate Pearson's correlation coefficients and generate a scatterplot of overlapping intensities where the darker pixels represent less frequent occurrences of pixel intensity, while yellow colors indicate a higher frequency of colocalization. Graphs for correlation coefficient over time lapse for formulation effects were plotted by importing the tabular results from ImageJ into GraphPad Prism 7.0 for data analysis.

\section{In vitro cytotoxicity study}

Cytotoxicity was evaluated by a growth inhibition assay, using 3-(4,5-dimethylthiazol-2-yl)-2,5-diphenyltetrazolium bromide staining as end point. Cells were seeded into 96 -well plates (800 cells/well) and cultured at $37^{\circ} \mathrm{C}, 5 \%$ $\mathrm{CO}_{2}$ for $24 \mathrm{~h}$. Then, the cells were exposed with a concentration series of blank liposomes, free drug or drug-loaded CL-PEG-pSL or PEG-pSL. After 24-h drug exposure, cells were washed and grown for $72 \mathrm{~h}$ before staining with 3(4,5-dimethylthiazol-2-yl)-2,5-diphenyltetrazolium bromide. The drug concentrations causing 50\% inhibition of viable cell density with respect to nontreated controls $\left(\mathrm{IC}_{50}\right)$ and the $95 \% \mathrm{CI}$ were calculated using a four-parameter logistic regression by GraphPad Prism 7.0 (GraphPad Software, USA).

\section{Pharmacokinetic \& biodistribution studies}

Sprague-Dawley rats (weighing $200 \pm 5 \mathrm{~g}$ ) and CD-1 nude mice (weighing $20 \pm 2 \mathrm{~g}$ ) were obtained from the Vernon Jansen Unit, the University of Auckland (Auckland, New Zealand). Pharmacokinetic and biodistribution studies were carried out under protocols approved by the Animal Ethics Committee of The University of Auckland (ethics approval no. 001593 and 001880 , respectively).

To determine the long-circulation property pharmacokinetics of gemcitabine formulations were studied in Sprague-Dawley rats. Animals were randomized to three groups: CL-PEG-pSL $(n=6)$, PEG-pSL $(n=4)$ and freedrug solution in PBS, pH $7.4(\mathrm{n}=4)$. Each formulation $(0.2 \mathrm{mg}$ gemcitabine $/ \mathrm{ml})$ was injected intravenously into the tail vein at $1 \mathrm{mg} / \mathrm{kg}$ body weight. Blood samples (100-200 $\mu \mathrm{l})$ were collected from the tail vein at various time intervals. Furthermore, a high dose of $5 \mathrm{mg} / \mathrm{kg}$ was injected to investigate dose dependence of the pharmacokinetics of the CL-PEG-pSL. Samples were prepared for analysis as per our previously reported method [26].

Gemcitabine biodistribution was evaluated in tumor-bearing CD-1 nude mice. $5 \times 10^{6} \mathrm{MIA}$ PaCa-2 cells (suspended in 1:1 PBS:Matrigel) were subcutaneously implanted into the hind flank of the mice. When tumors reached approximately $300-400 \mathrm{~mm}^{3}$, mice were randomized to three treatment groups (three mice/group) and injected with free gemcitabine or gemcitabine-loaded PEG-pSL and CL-PEG-pSL at a dose of $16 \mathrm{mg} / \mathrm{kg}$ body weight via the tail vein. Animals were culled $4 \mathrm{~h}$ after dosing and blood, major organs (liver, kidney, spleen, heart) and tumors were harvested and stored on dry ice for HPLC analysis. In addition, biodistribution after $12 \mathrm{~h}$ of dosing was evaluated in tumor-free CD-1 nude mice $(n=3)$. Plasma samples were treated in a similar way to the aforementioned pharmacokinetics study. Organs were added to acetonitrile $(0.2 \mathrm{~g}: 1 \mathrm{ml})$ and homogenized by a 
(A)
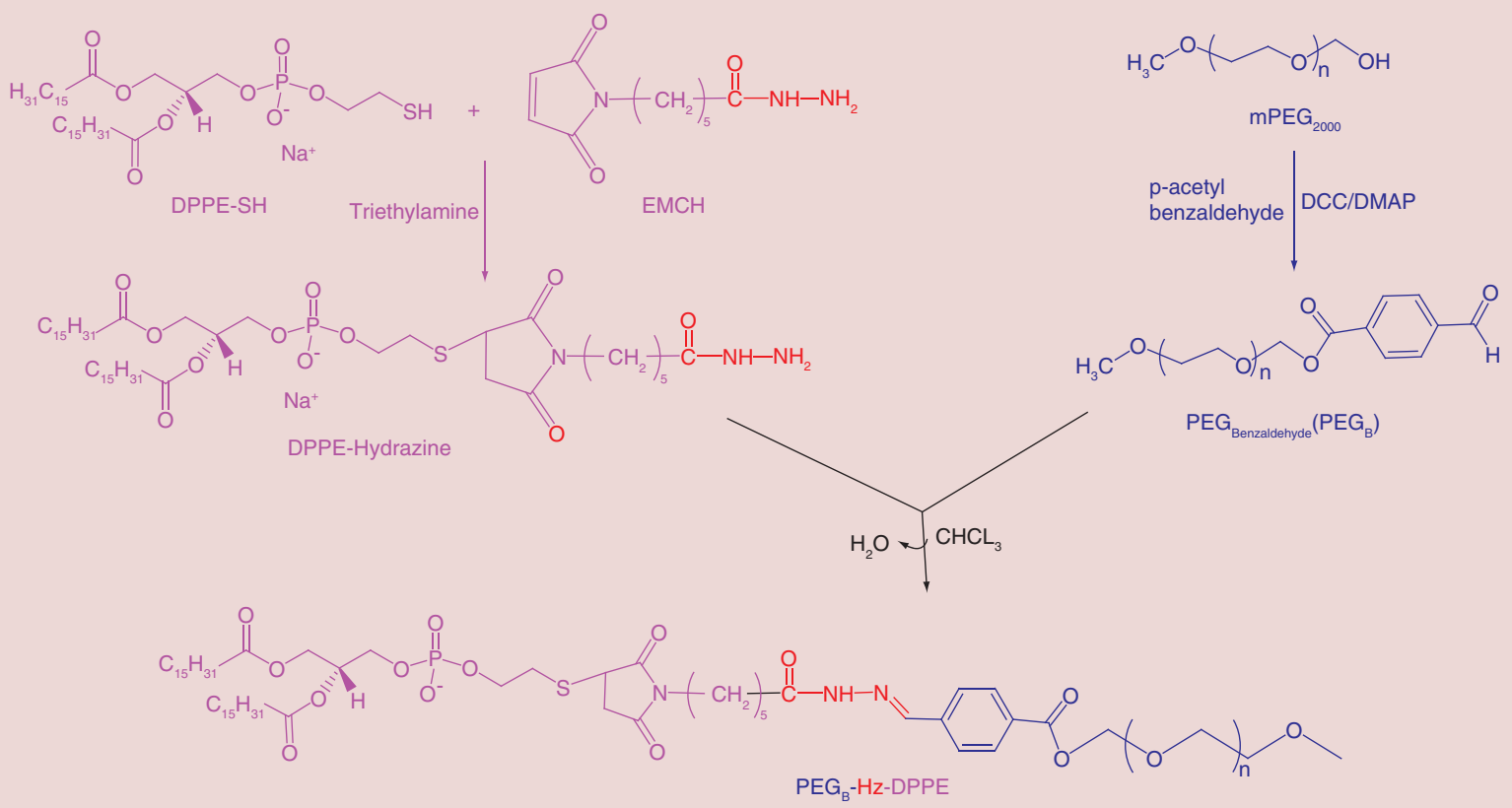

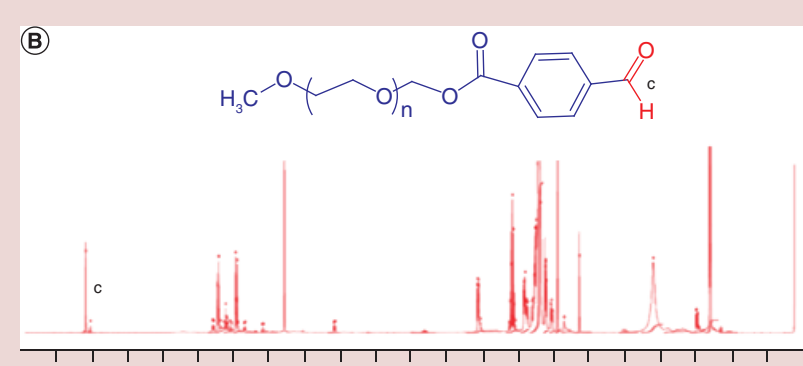

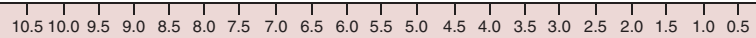

ppm

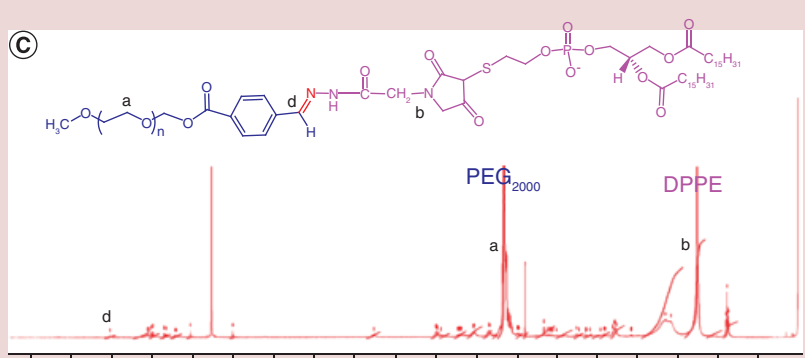

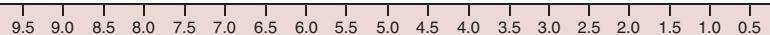

ppm

Figure 1. Synthesis and characterization of $P_{E} G_{B}-H z-D P P E .(A)$ Scheme of synthesis of $P E G_{B}-H z-D P P E$, typical ${ }^{1} H$ NMR spectrum of (B) $\mathrm{PEG}_{\mathrm{B}}$ and (C) $\mathrm{PEG}_{\mathrm{B}}-\mathrm{Hz}-\mathrm{DPPE}$.

DCC: N,N'-Dicyclohexylcarbodiimide; DMPA: 4-Dimethylaminopyridine; DPPE: 1,2-Dipalmitoyl-sn-glycero-3-phosphoethanolamine; Hz: Hydrazone; PEG: Poly(ethene glycol).

tissue dissociator (gentle MACS Dissociator, Miltenyi Biotech) at $2000 \times \mathrm{g}$ for $1.5 \mathrm{~min}$ and samples were then centrifuged at 10,000 $\times \mathrm{g}$. The supernatant was evaporated, and the residue redissolved in $50 \mu \mathrm{l} \mathrm{Milli} \mathrm{Q}$ water and gemcitabine concentrations were determined by HPLC. The limit of detection was $0.05 \mu \mathrm{g} / \mathrm{ml}$ for both plasma and tissues and the extraction recovery for drug spiked blanks was observed as more than $85 \%$.

\section{Data \& statistical analysis}

The gemcitabine pharmacokinetic profiles were fitted to a noncompartmental model using Phoenix WinNonlin 8.1 (Certara, NJ, USA) to obtain the pharmacokinetic parameters. Data were analyzed by one-way analysis of variance (ANOVA) with Tukey's multiple comparisons test using GraphPad Prism 7.01 (GraphPad Software Inc., CA, USA). The level of significance for all statistical analysis was set at 0.05 .

\section{Results}

Synthesis of PEGB-Hz-DPPE polymer

The synthetic routes and the molecular structure of $\mathrm{PEG}_{\mathrm{B}}-\mathrm{Hz}-\mathrm{DPPE}$ are shown in Figure 1A. The structure of the polymer was confirmed by ${ }^{1} \mathrm{H}$ NMR spectroscopy (Figure 1B \& C). The detailed peak analysis of the polymer was 
(A)

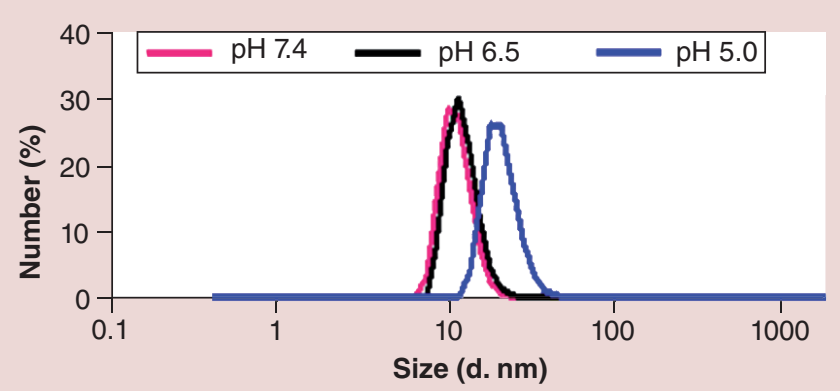

(C)

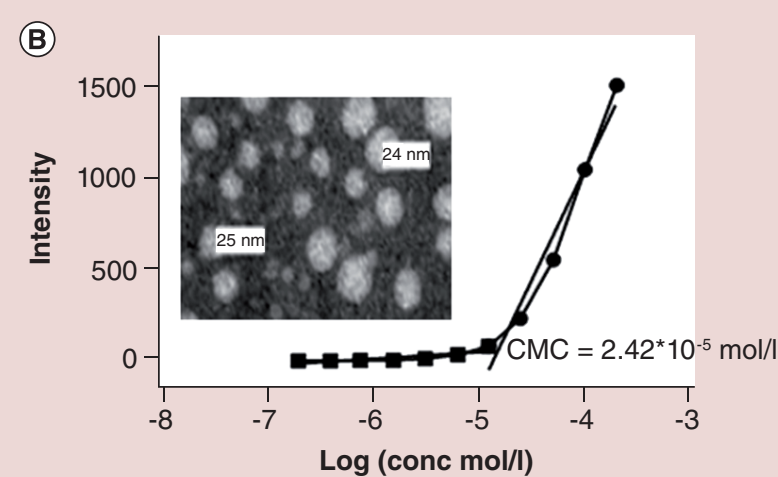

$\log ($ conc $\mathrm{mol} / \mathrm{l})$

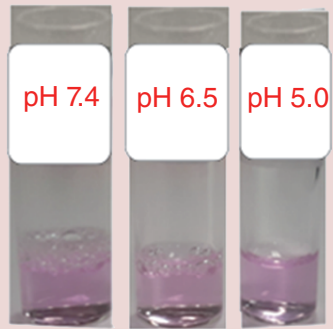

$\mathrm{Oh}$

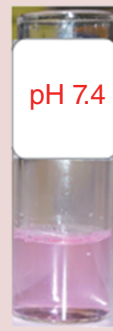

$2 \mathrm{~h}$

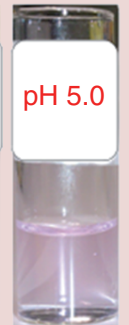

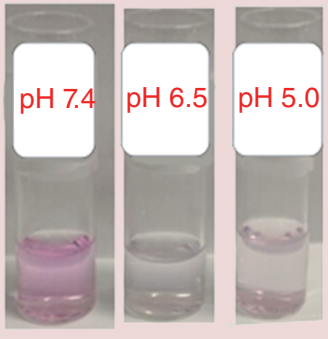

$24 \mathrm{~h}$

Figure 2. Size and pH-sensitivity of $\mathrm{PEG}_{\mathrm{B}}-\mathrm{Hz}$-1,2-dipalmitoyl-sn-glycero-3-phosphoethanolaminepolymeric micelles. (A) Dynamic light scattering showing a shift in particle size after $2 \mathrm{~h}$ incubation at $\mathrm{pH} 6.5$ and 5 in contrast to that at pH 7.4, (B) morphology of the polymeric micelles and critical micellar concentration of $\mathrm{PEG}_{\mathrm{B}}-\mathrm{Hz}-1,2$-dipalmitoyl-sn-glycero-3-phosphoethanolamine and (C) visual observation of changes in color of micelle solutions with $\mathrm{pH}$, solutions at low $\mathrm{pH}$ appeared colorless due to their $\mathrm{pH}$-dependent destabilization of polymeric micelles, causing release and precipitation of Nile Red dye.

CMC: Critical micellar concentration.

displayed as chemical shift values $\delta$ of $0-3$ ppm (peak b, the methyl and methylene group of DPPE), 3.09-4 (peak a, $P_{E} G_{B}$ ) and 7.9-8.0 (p-phenyl ring of the benzaldehyde). Furthermore, after the coupling reaction between $P^{-1} G_{B}$ and DPPE-Hz, the characteristic proton signal of the aldehyde group ( $\delta 10.5 \mathrm{ppm}$, peak c) derived from $\mathrm{PEG}_{\mathrm{B}}$ disappeared completely, and the proton signal of the imine group ( $\delta 8.50 \mathrm{ppm}$, peak $\mathrm{d})$ appeared in the ${ }^{1} \mathrm{H}$ NMR spectrum of the conjugate. The NMR integrals of $\mathrm{PEG}_{\mathrm{B}}-\mathrm{Hz}-\mathrm{DPPE}$ polymer were: $0.84-0.86(\mathrm{~m}, 6 \mathrm{H}), 1.23-1.30$ $(\mathrm{m}, 45 \mathrm{H}), 1.63(\mathrm{~s}, 40 \mathrm{H}), 1.96-2.00(\mathrm{~m}, 2 \mathrm{H}), 2.15(\mathrm{~s}, 4 \mathrm{H}), 2.24-2.28(\mathrm{q}, 5 \mathrm{H}), 2.53-2.55(\mathrm{t}, 2 \mathrm{H}), 3.55-3.66(\mathrm{~m}$, 232H), 3.75-3.76 (s, 3H), 3.98-4.04 (q, 3H), 4.16-4.20 (m, 1H), 4.39-4.41 (s, 4H), 5.2 (s, 2H), 7.64-7.66 (d, $2 \mathrm{H}), 7.85(\mathrm{~d}, 2 \mathrm{H}), 8.0(\mathrm{~d}, 2 \mathrm{H}), 8.5(\mathrm{~s}, 1 \mathrm{H})$

\section{Characterization of $\mathrm{PEG}_{\mathrm{B}}-\mathrm{Hz}-\mathrm{DPPE}$}

The synthesized $\mathrm{PEG}_{\mathrm{B}}-\mathrm{H}_{\mathrm{Z}}$-DPPE spontaneously aggregated into polymeric micelles in aqueous media because of the presence of the hydrophilic PEG and hydrophobic DPPE domains. The obtained micelle dispersion in PBS (0.01 M, pH 7.4) exhibited an average particle size of $40 \mathrm{~nm}$ with a narrow unimodal size distribution at physiological $\mathrm{pH}$ (Figure 2A). Monodispersed spherical $\mathrm{PEG}_{\mathrm{B}}-\mathrm{Hz}-\mathrm{DPPE}$ micelles were confirmed by cryoTEM with an average size of $25-35 \mathrm{~nm}$. The CMC of the polymer was determined as $24.2 \mu \mathrm{M}$ by fluorescence spectrometry as the turning point of releasing the incorporated Nile Red (Figure 2B).

In contrast to the micelles at $\mathrm{pH} 7.4$, which were stable, a rapid increase in the size of the micelles was observed postincubation at $\mathrm{pH} 5.0$ for $2 \mathrm{~h}$, whereas size remained the same after incubation at $\mathrm{pH} 6.5$ for the same period (Figure 2A). Confirming the above results, Nile Red assay also revealed that micelles entrapping Nile Red in their core exhibited strong fluorescence intensity at $\mathrm{pH} 7.4$, which remained the same after $24 \mathrm{~h}$. However, after incubation of micelles for $2 \mathrm{~h}$ at $\mathrm{pH} 6.5\left(\mathrm{pH}_{\mathrm{ex}}\right)$ and $5.0\left(\mathrm{pH}_{\mathrm{en}}\right)$, a sharp reduction in fluorescence intensity was observed. The color completely disappeared from the micellar solution after $24 \mathrm{~h}$ with a visible amount of Nile Red released to the top of the solution, indicating the ability of micelles to undergo $\mathrm{pH}$-triggered destabilization 
(A)

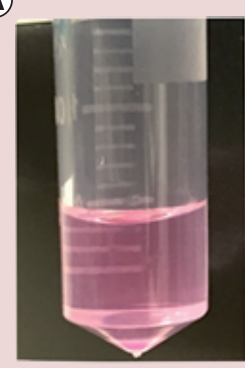

PEG-Hz-DPPE micelles
(B)

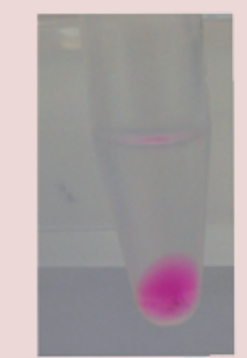

Liposomes post insertion with PEGB-Hz-DPPE

micelles

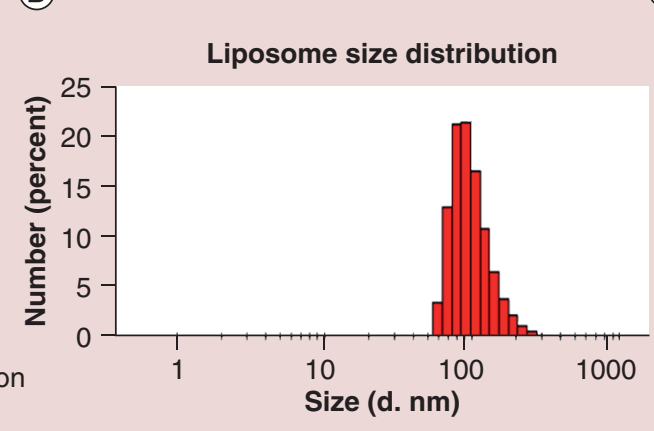

(C)

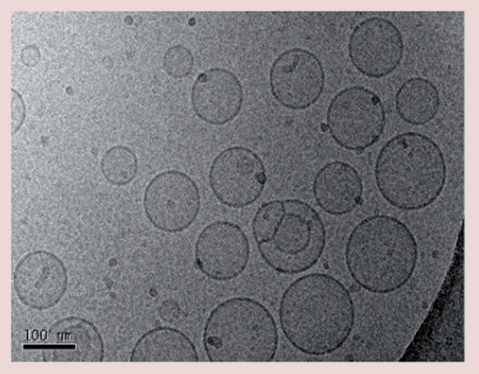

Figure 3. Characterization of $\mathrm{PEG}_{\mathrm{B}}-\mathrm{Hz}-\mathrm{DPPE}-\mathrm{modified}$ liposomes. (A) Polymer insertion efficiency: $\mathrm{PEG}_{\mathrm{B}}-\mathrm{Hz}_{\mathrm{H}}-\mathrm{DPPE}$ micelles entrapping Nile red dye before (left) and postincubation with 1,2-dioleoyl-sn-glycero-3-phosphoethanolamine/cholesteryl hemisuccinate liposomes and ultracentrifugation to remove free Nile red micelles, supernatant appeared clear showing more Nile red micelles were inserted in the liposomal pellet (right); (B) size distribution and (C) morphology of cleavable-PEG-pH-sensitive liposomes.

DPPE: 1,2-Dipalmitoyl-sn-glycero-3-phosphoethanolamine; Hz: Hydrazone; PEG: Poly(ethylene glycol).

\begin{tabular}{|c|c|c|c|c|c|}
\hline Formulation & Time (month) & Size $(\mathrm{nm})$ & PDI & Zeta potential (mV) & $\begin{array}{l}\text { Gemcitabine entrapped in } \\
\text { liposomes (\%) }\end{array}$ \\
\hline \multirow[t]{3}{*}{ CL-PEG-pSL } & 0 & $143 \pm 0.62$ & $0.07 \pm 0.02$ & $-9.5 \pm 1.0$ & $100.0 \pm 0.6$ \\
\hline & 1 & $142 \pm 0.36$ & $0.06 \pm 0.01$ & $-9.8 \pm 1.2$ & $98.5 \pm 1.0$ \\
\hline & 2 & $146 \pm 0.12$ & $0.09 \pm 0.03$ & $-9.4 \pm 1.5$ & $91.3 \pm 1.0$ \\
\hline \multirow[t]{3}{*}{ PEG-pSL } & 0 & $142 \pm 0.10$ & $0.04 \pm 0.01$ & $-7.6 \pm 1.4$ & $100.0 \pm 0.2$ \\
\hline & 1 & $141 \pm 0.14$ & $0.08 \pm 0.02$ & $-8.2 \pm 1.1$ & $98.2 \pm 0.5$ \\
\hline & 2 & $144 \pm 0.11$ & $0.05 \pm 0.02$ & $-8.2 \pm 1.3$ & $92.5 \pm 1.0$ \\
\hline
\end{tabular}

(Figure 2C). Additionally, it was found that the color reduction appeared to accelerate with the decrease of $\mathrm{pH}$ from 6.5 to 5.0 .

\section{Characterization of PEGylated liposomes}

Following incubation of empty liposomes with $\mathrm{PEG}_{\mathrm{B}}-\mathrm{Hz}-\mathrm{DPPE}$ micelles containing Nile red, the resulting liposomes in pellet form (obtained by ultracentrifugation) showed pink color while the supernatant appeared colorless. This infers that the lipophilic Nile red had been transferred into liposomes. Partition of Nile Red from the lipid cores of thermodynamically stable micelles to liposomes is almost unlikely, unless migration of DPPE-Hz-PEG to the liposome bilayers that caused micelles leakage of Nile Red. Based on this assumption, the insertion efficiency of the polymer was estimated to be more than $95 \%$ based on visual observation (Figure 3A). The average size of the resulting CL-PEG-pSL was $121.3 \pm 0.6 \mathrm{~nm}$ (Figure 3B) with a round and unilamellar structure (Figure 3C). After PEGylation, along with an increase in their size, CL-PEG-pSL exhibited a decrease in the zeta potential. Furthermore, NMR spectrum of CL-PEG-pSL showed the PEG integrals at chemical shift value 3.09-4, similar to the polymer, confirming the insertion of the polymer into liposomes (Supplementary data).

The liposomes were then loaded with gemcitabine with an EE of $37.0 \pm 1.0 \%$, and a high DL of $4 \% \mathrm{w} / \mathrm{w}$. Drug-loaded CL-PEG-pSL and PEG-pSL showed an average size of $143 \pm 0.62$ and $142 \pm 0.10 \mathrm{~nm}$, respectively (Table 1). The liposomes showed good stability over 2 months when stored at $4{ }^{\circ} \mathrm{C}$ as liposome pellets (Table 1 ). Doxorubicin-loaded liposomes showed an EE of $99.0 \pm 0.5 \%$, and DL of $7 \% \mathrm{w} / \mathrm{w}$ as determined by HPLC.

\section{pH-Responsive drug release}

There was a $\mathrm{pH}$ dependence of drug release from both PEG-pSL and CL-PEG-pSL particularly at $\mathrm{pH} 5\left(\mathrm{pH}_{\mathrm{en}}\right.$; Figure 4). At $\mathrm{pH} 7.4$, the cumulative release of gemcitabine from PEG-pSL and CL-PEG-pSL was similar (30\%) 
(A)

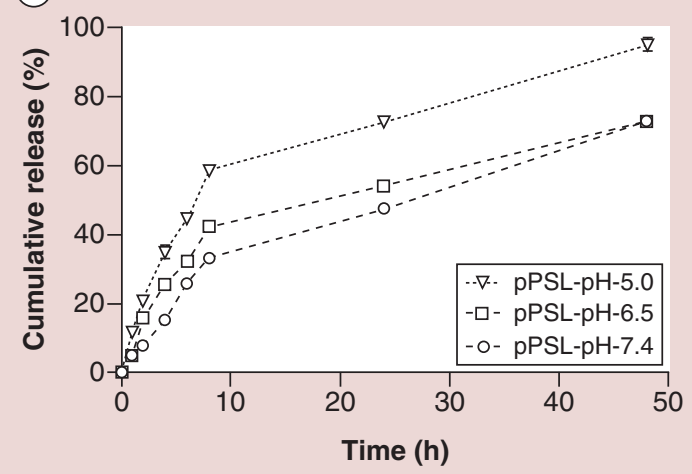

(B)

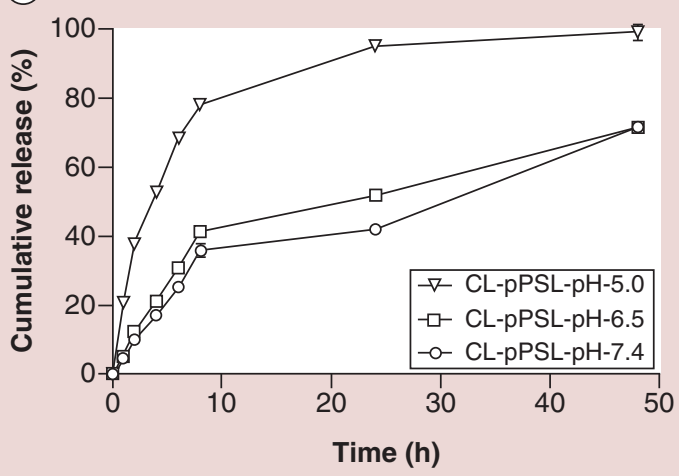

Figure 4. pH-responsive gemcitabine release profiles. (A) Poly(ethylene glycol)-pSL and (B) CL-poly(ethylene glycol)-pSL at pH 5.5, 6.5 and 7.4 at $37^{\circ} \mathrm{C}$ (mean \pm standard deviation, $\mathrm{n}=3$ ). $\mathrm{CL}$ : Cleavable; pSL: pH-sensitive liposome.

during the first $8 \mathrm{~h}$. Between CL-PEG-pSL and PEG-pSL, the overall release profiles of (Figure 4) are similar both at $\mathrm{pH} 7.4$ and 6.5 with $f_{2}$ factors of 79.1 and 75.5 , respectively. As $\mathrm{pH}$ of the release medium was reduced to 5.0, the release rate from both liposomes increased, 78.4\% from CL-PEG-pSL, and 58.5\% from PEG-pSL, leading to an $f_{2}$ value of 38.9, which indicates that the release profiles from the two liposomes were not similar.

\section{In vitro cellular uptake \& endosome escape studies}

Cellular uptake in MIA PaCa-2 cells was indicated from the red fluorescence of Nile Red-labeled liposomes, and endosome escape was denoted by the green fluorescence of calcein. A high concentration of calcein $80 \mathrm{mM}$ (selfquenched) was encapsulated in the liposomal cores, which become visible only after it is released from liposomes and is diluted to its dequenched concentration $(<10 \mathrm{mM})$ [34]. It was evident that no significant difference was observed in red fluorescence intensity between CL-PEG-pSL and PEG-pSL (Figure 5A), indicating that both liposomes were being taken up to the same extent. However, the green channel clearly distinguished the two formulations with a threefold higher fluorescence intensity for CL-PEG-pSL than for PEG-pSL at $30 \mathrm{~min}$. This indicated that CL-PEG-pSL underwent a rapid endosomal escape, thus readily releasing the self-quenched the encapsulated calcein from the liposomal cores into the cytoplasm. ImageJ quantification analysis (Figure 5B) showed that after $1 \mathrm{~h}$, the fluorescence intensity of calcein increased for both liposomes, while CL-PEG-pSL still displayed 1.8-fold higher intensity than PEG-pSL. Following $2 \mathrm{~h}$ incubation, cells treated with CL-PEG-pSL showed homogenous distribution of calcein around the nucleus. In contrast, PEG-pSL-treated cells exhibited only a weak punctate distribution of fluorescence, suggesting some of the PEG-pSL were still entrapped in the endosomal compartments.

\section{Subcellular localization of CL-PEG-pSL \& PEG-pSL by live cell imaging}

Interestingly, live cell imaging showed that the LysoTracker Deep Red (green color)-labeled vesicles (late endosomes or lysosomes) in MIA PaCa-2 cells were initially concentrated around the center and distributed around the nucleus, with only a few found around the periphery of the cell (Figure 6A \& B). After $20 \mathrm{~min}$ of exposure to cells, Rh-PE-labeled CL-PEG-pSL (red color) were found to be colocalized in endo/lysosomal regions, which increased over time. By the end of $1 \mathrm{~h}$, bright red fluorescence of CL-PEG-pSL was homogenously distributed around the nucleus, indicating content release into cytoplasm. In contrast, PEG-pSL showed a weak red fluorescence signal and by the end of $1 \mathrm{~h}$ only the punctate pattern was observed in the endo/lysosomal region. For both CL-PEG-pSL and PEG-pSL, with a progression in time from $5 \mathrm{~min}$ to $2 \mathrm{~h}$, the images show the trafficking of liposomes in the cell from being taken up into the cells (red fluorescence of liposomes spotted in green-stained cells), to fuse with endo/lysosomal membrane (red merging with green to form yellow), and to release into the cytoplasm around nucleus (reddish yellow).

Scatter plots of CL-PEG-pSL-treated cells displayed a brighter intensity of yellow pixels from 5 min, indicating the strong colocalization of Rh-PE (liposomes) with Lysotracker (endo/lysosomes regions). The yellow pixels are highest at $30 \mathrm{~min}$, and started to decline after $1 \mathrm{~h}$, probably due to dissipation of the liposomes from endo/lysosomes into 


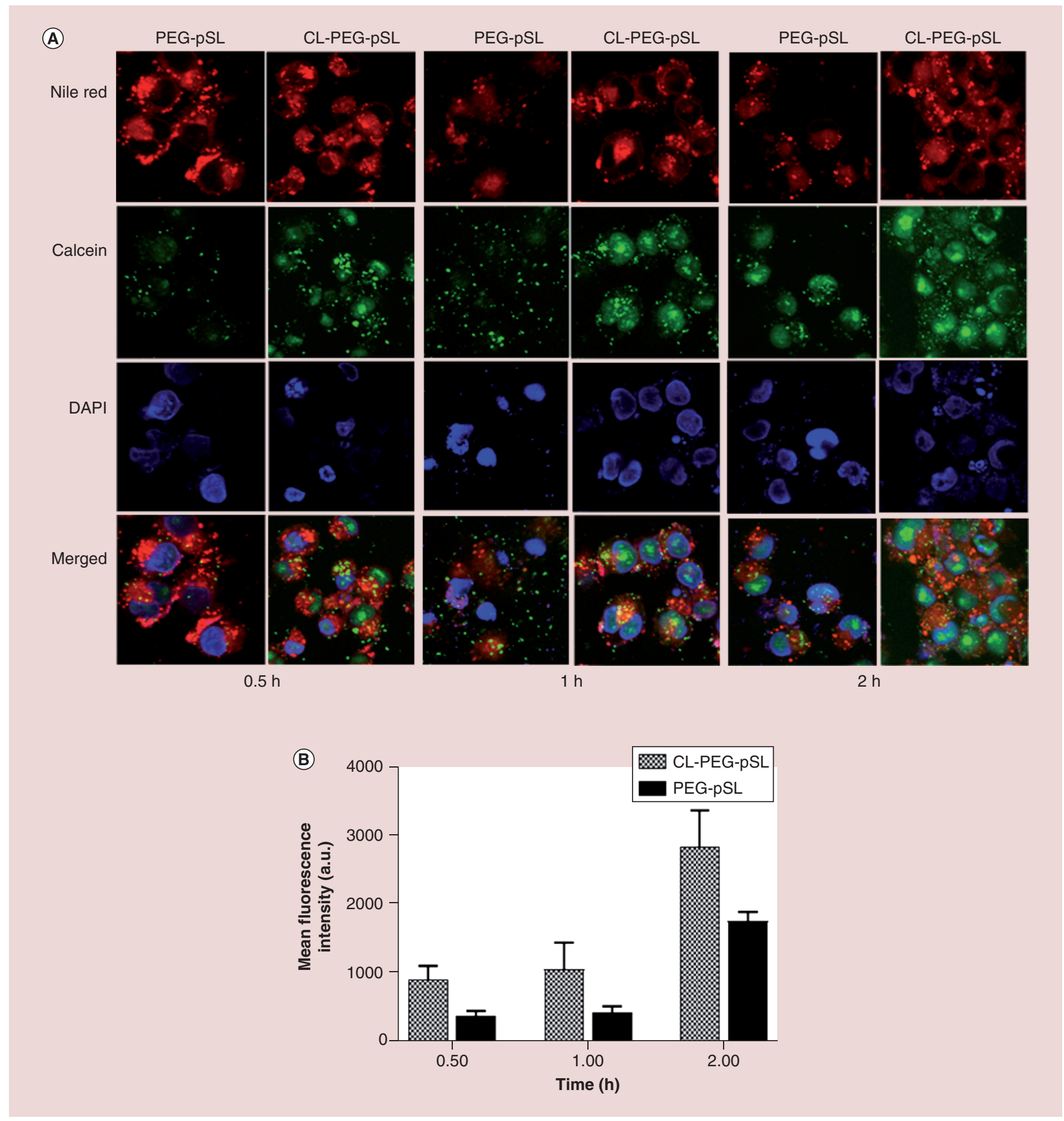

Figure 5. Cell uptake and endosomal escape abilities of PEG-pSL and CL-PEG-pSL. (A) CLSM images showing the cell uptake and endosome escape of dual-labeled PEG-pSL and CL-PEG-pSL in MIA PaCa-2 cell lines over $2 \mathrm{~h}$. The Nile Red-positive cells indicate cell uptake and calcein-positive indicate endosomal escape. DAPI (shown here as blue) was used as the nuclear stain. (B) Quantification of fluorescence intensity of calcein (green channel) from each liposomal formulation as an indicator of endosomal escape in MIA PaCa-2 cells, processed by ImageJ. Fluorescence intensity is expressed in AU. The values represent mean \pm standard deviation ( $n=3$ ). AU: Arbitrary units; CL: Cleavable; CLSM: Confocal laser scanning microscope; DAPI: 4',6-diamidino-2-phenylindole; PEG: Poly(ethylene glycol); pSL: pH-sensitive liposome. 


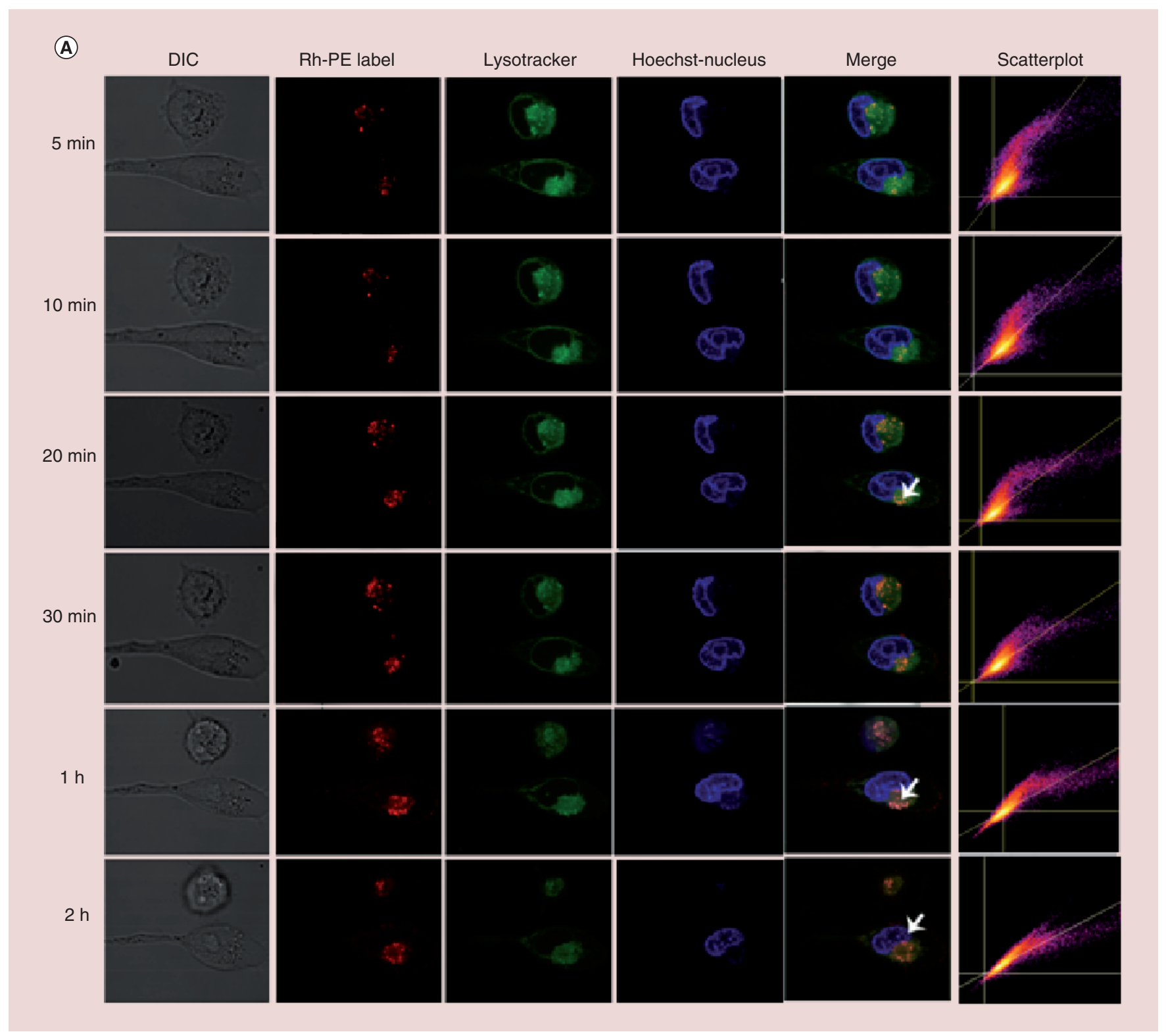

Figure 6. Subcellular localization of cleavable-PEG-pH-sensitive liposomes and PEG-pH-sensitive liposomes by live cell imaging. (A) Live cell imaging showing the intracellular trafficking of rhodamine-labeled CL-PEG-pSL over a 2-h time period. DIC (gray images) was used to monitor the cell morphology throughout the process. Rh-PE was used to label the liposomes. The LysoTracker Deep Red (shown here as the green counterstain) was used to label the late endo/lysosomal regions of the cell. Hoechst 33342 (shown here as blue) was used as the nuclear stain. ImageJ was used to generate a scatter plot of pixel intensities using the merged Lysotracker and Rh-PE channels. The cleavable-PEG-pSL displayed a clearly evident colocalization after $20 \mathrm{~min}$ incubation time indicating their fusion with endo/lysosomal membrane (red liposomes merging with green endosomal staining indicated by the arrow). After $1 \mathrm{~h}$ of incubation, the disseminated dots showed a brighter continuous pattern, possibly indicating rapid leakage from the endosomes into the cytoplasm around nucleus (bright red dots around the nuclear region as indicated by the arrows). Scatter plots displayed a brighter intensity of yellow pixels from $5 \mathrm{~min}$, which is highest at $30 \mathrm{~min}$, however, the region of yellow intensity started to decline after $1 \mathrm{~h}$, probably due to dissipation of the liposomes from endo/lysosomes into cytoplasm. (B) Live cell imaging snapshotted at different time points showing the intracellular trafficking of rhodamine-labeled PEG-pSL over a 2-h time period. DIC was used to monitor the cell morphology throughout the process. $\mathrm{Rh}$-PE was used to label the liposome membranes. The LysoTracker Deep Red (shown here as the green counterstain) was used to label the late endo/lysosomal regions of the cell. Hoechst 33342 (shown here as blue) was used as the nuclear stain. Images displayed a weak and fine punctuate colocalization of PEG-pSL in endo/lysosomal region after 20 min of incubation time (indicated by the arrow). However, the PEG-pSL were more evident at the end of $1 \mathrm{~h}$ and could be seen to have undergone fusion with the endo/lysosomal membrane (red liposomes merging with green endosomal staining are indicated by the arrow) and at $2 \mathrm{~h}$ the PEG-pSL exhibited a visible leakage from the endosomes into the cytoplasm around the nucleus (bright red dots around the nuclear region are indicated by the third arrow). The scatter plot of pixel intensities was generated using ImageJ by merging Lysotracker and Rh-PE channels showing a low frequency of colocalization of liposomes and endo/lysosomes (dark pixels), however, with an increase in the yellow pixels with time. CL: Cleavable; DIC: Differential interference contrast; PEG: Poly(ethene glycol); pSL: pH-sensitive liposome; Rh-PE: 1,2-Dipalmitoyl-sn-glycero-3-phosphoethanolamine- $N$-(lissamine rhodamine B sulfonyl). 


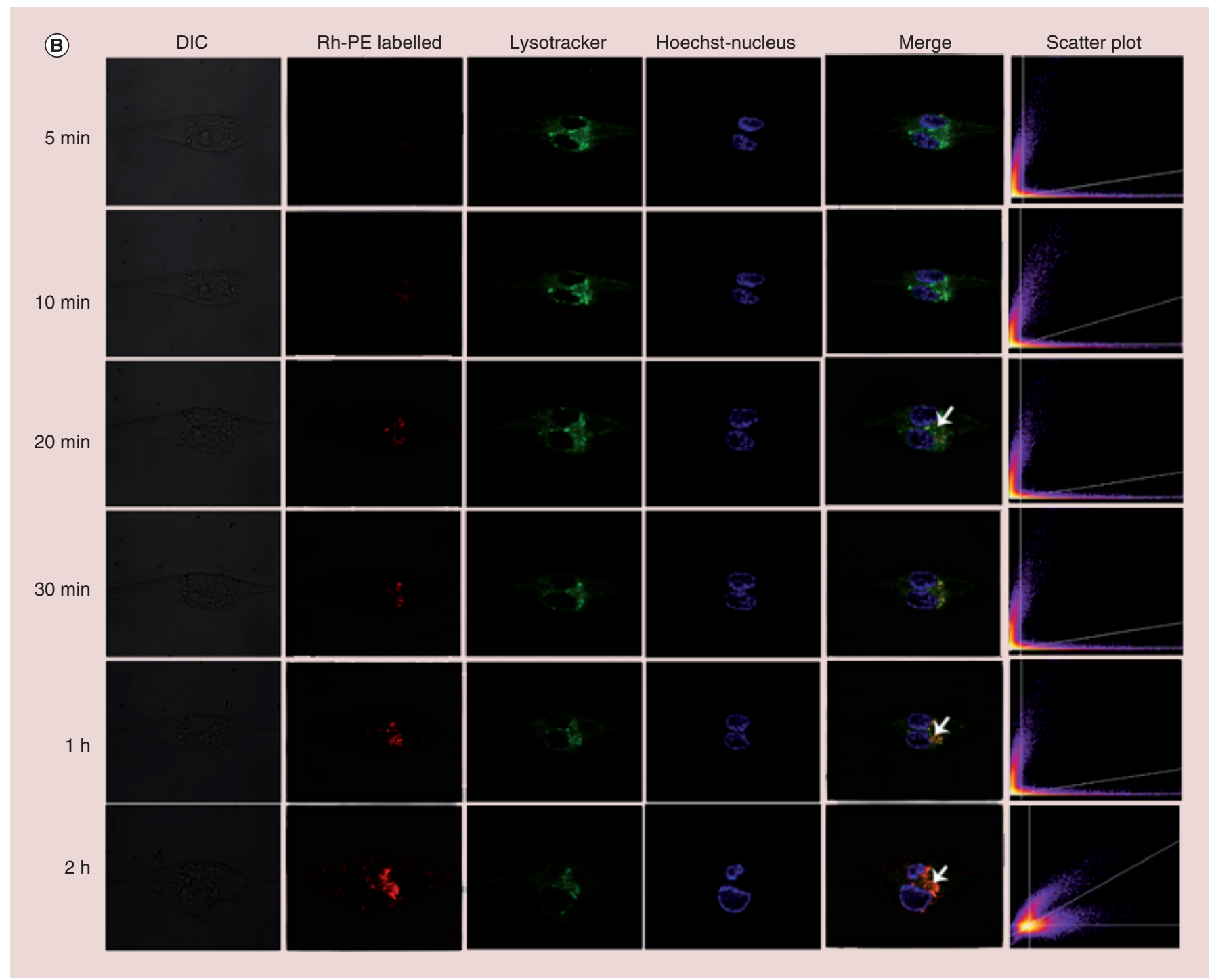

Figure 6. Subcellular localization of cleavable-PEG-pH-sensitive liposomes and PEG-pH-sensitive liposomes by live cell imaging (cont.). (A) Live cell imaging showing the intracellular trafficking of rhodamine-labeled CL-PEG-pSL over a 2-h time period. DIC (gray images) was used to monitor the cell morphology throughout the process. Rh-PE was used to label the liposomes. The LysoTracker Deep Red (shown here as the green counterstain) was used to label the late endo/lysosomal regions of the cell. Hoechst 33342 (shown here as blue) was used as the nuclear stain. ImageJ was used to generate a scatter plot of pixel intensities using the merged Lysotracker and Rh-PE channels. The cleavable-PEG-pSL displayed a clearly evident colocalization after 20 min incubation time indicating their fusion with endo/lysosomal membrane (red liposomes merging with green endosomal staining indicated by the arrow). After $1 \mathrm{~h}$ of incubation, the disseminated dots showed a brighter continuous pattern, possibly indicating rapid leakage from the endosomes into the cytoplasm around nucleus (bright red dots around the nuclear region as indicated by the arrows). Scatter plots displayed a brighter intensity of yellow pixels from $5 \mathrm{~min}$, which is highest at $30 \mathrm{~min}$, however, the region of yellow intensity started to decline after $1 \mathrm{~h}$, probably due to dissipation of the liposomes from endo/lysosomes into cytoplasm. (B) Live cell imaging snapshotted at different time points showing the intracellular trafficking of rhodamine-labeled PEG-pSL over a 2-h time period. DIC was used to monitor the cell morphology throughout the process. Rh-PE was used to label the liposome membranes. The LysoTracker Deep Red (shown here as the green counterstain) was used to label the late endo/lysosomal regions of the cell. Hoechst 33342 (shown here as blue) was used as the nuclear stain. Images displayed a weak and fine punctuate colocalization of PEG-pSL in endo/lysosomal region after 20 min of incubation time (indicated by the arrow). However, the PEG-pSL were more evident at the end of $1 \mathrm{~h}$ and could be seen to have undergone fusion with the endo/lysosomal membrane (red liposomes merging with green endosomal staining are indicated by the arrow) and at $2 \mathrm{~h}$ the PEG-pSL exhibited a visible leakage from the endosomes into the cytoplasm around the nucleus (bright red dots around the nuclear region are indicated by the third arrow). The scatter plot of pixel intensities was generated using ImageJ by merging Lysotracker and Rh-PE channels showing a low frequency of colocalization of liposomes and endo/lysosomes (dark pixels), however, with an increase in the yellow pixels with time. CL: Cleavable; DIC: Differential interference contrast; PEG: Poly(ethene glycol); pSL: pH-sensitive liposome; Rh-PE: 1,2-Dipalmitoyl-sn-glycero-3-phosphoethanolamine- $N$-(lissamine rhodamine B sulfonyl). 
Figure 7. Pearson's correlation coefficients for colocalization of Lysotracker (green indicating endo/lysosomal regions) and rhodamine (red indicating liposomes) in MIA PaCa-2 cells for CL-PEG-pH-sensitive liposomes and PEG-pH-sensitive liposomes. CL: Cleavable; PEG: Poly(ethene glycol); pSL: pH-sensitive liposome.

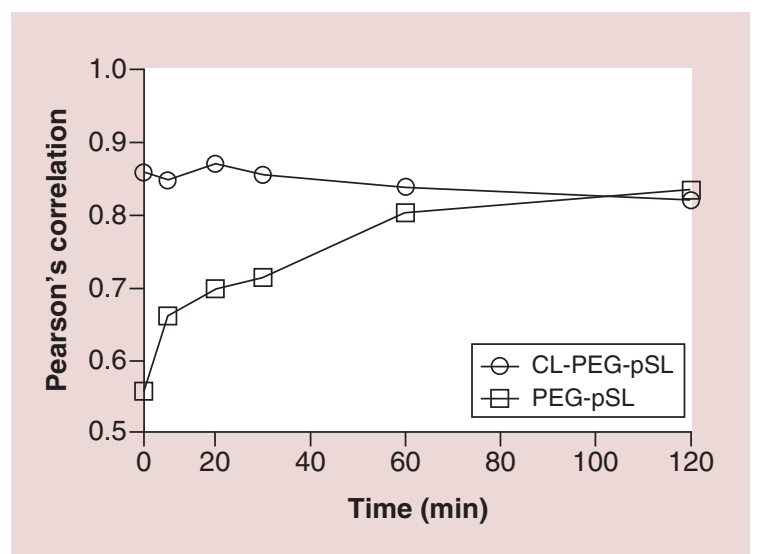

Table 2. Cytotoxicity measured as $\mathrm{IC}_{50}$ values $(\mathrm{nM})$ of gemcitabine or doxorubicin formulations as free drug, drug-loaded PEG-pH-sensitive liposomes and cleavable-PEG-pH-sensitive liposomes.

\begin{tabular}{|llc|} 
& Gemcitabine formulations to MIA PaCa-2 & Doxorubicin formulations to U-87 MG \\
\hline Free drug & $57.2 \pm 9.7$ & $179.3 \pm 67.9$ \\
\hline CL-PEG-pSL & $82.5 \pm 4.5$ & $91.9 \pm 8.3$ \\
\hline PEG-pSL & $96.0 \pm 19.8$ & $146.9 \pm 36.1$ \\
\hline
\end{tabular}

MIA PaCa-2 cells were exposed to gemcitabine formulations for $24 \mathrm{~h}$ followed by a 48 -h growth period. U-87 MG cells were exposed to doxorubicin formulations for $18 \mathrm{~h}$ followed by a 54-h growth period. Values represent the mean and standard deviation of three separate experiments.

CL: Cleavable; PEG: Poly(ethene glycol); pSL: pH-sensitive liposome.

cytoplasm. In contrast, PEG-pSL-treated cells represent darker pixels, indicating a low frequency of colocalization of liposomes and endo/lysosomes. However, there is a trend of increase in the yellow pixels with time.

The Pearson's correlation coefficients (r) over time were showed in Figure 7, where +1 indicates positive linear correlation and -1 indicates negative linear anticorrelation with zero if uncorrelated, further revealed the rapid accumulation and release of the CL-PEG-pSL into endo/lysosomal compartments over PEG-pSL. The $r$ values of the CL-PEG-pSL rapidly reached 0.85 within $5 \mathrm{~min}$ with further slight increase up to $30 \mathrm{~min}$ before starting to decline, indicating their rapid accumulation followed by diffusing out from endo/lysosomal lumens. A different pattern for PEG-pSL was observed, with a much lower $r$ value of 0.5 starting at 5 min, which gradually increased 0.8 over $1 \mathrm{~h}$ time period, indicating their slower accumulation into endo/lysosomal regions.

\section{In vitro cytotoxicity study}

As shown in Table 2, gemcitabine and doxorubicin formulations showed concentration-dependent cytotoxic effects in MIA PaCa-2 and U-87 MG cells. As indicated in their $\mathrm{IC}_{50}$ values, gemcitabine-loaded CL-PEG-pSL were slightly more cytotoxic than gemcitabine-loaded PEG-pSL $(\mathrm{p}<0.01)$. However, both liposomal formulations were less cytotoxicthan the free drug ( $\mathrm{p}<0.01$ ). In comparison, to U-87 MG cells, doxorubicin-loaded CL-PEG-pSL showed significantly greater cytotoxicity than both doxorubicin-loaded PEG-pSL and free doxorubicin $(\mathrm{p}<0.01$ in both cases).

In both cases, blank liposomes (diluted to lipid concentrations equivalent to the gemcitabine-loaded liposomes) caused negligible toxicity over 24 -h exposure periods (about $90 \%$ cell viability), indicating good biocompatibility of the liposomes.

\section{Pharmacokinetic \& biodistribution studies}

The plasma concentration curves (Figure 8A) show that both liposome formulations increased the retention time of the drugs in blood, whereas free gemcitabine was eliminated quickly. The pharmacokinetic parameters further confirmed this result (Table 3). Compared with the drug solution, both CL-PEG-pSL and PEG-pSL show significant reductions in gemcitabine plasma clearance and volume of distribution, and a six- to eightfold increase in the area under the curve $\left(\mathrm{AUC}_{0-\infty} ; \mathrm{p}<0.001\right)$. In addition, there was an approximately threefold increase in the elimination half-life $\left(\mathrm{T}_{1 / 2}\right)$ and an approximately 1.5 -fold increase in the mean residence time for both liposomes compared with the drug solution. Further study with an increase in the dose of CL-PEG-pSL from 1 to $5 \mathrm{mg} / \mathrm{kg}$ 


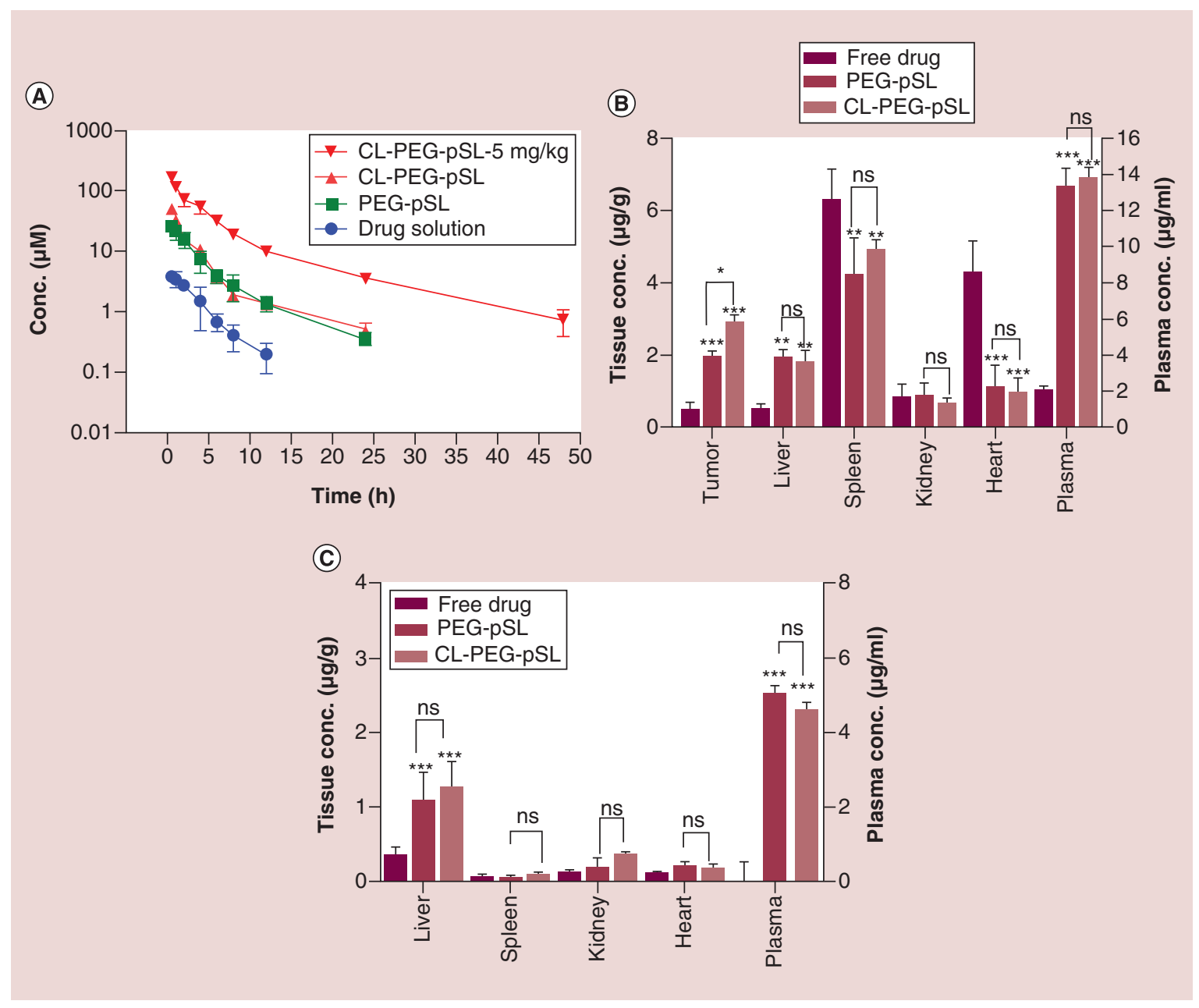

Figure 8. Plasma pharmacokinetics profiles and tissue biodistribution of various gemcitabine formulations. (A) Plasma pharmacokinetic profiles following intravenous injection to Sprague-Dawley rats at $1 \mathrm{mg} / \mathrm{kg}$ equivalent gemcitabine or $5 \mathrm{mg} / \mathrm{kg}(\mathrm{n}=4-6)$ (note this is the same reference for [26]); Concentration of gemcitabine in various tissues at $4 \mathrm{~h}$ (B) and $12 \mathrm{~h}$ (C) following a single injection of gemcitabine formulations, at gemcitabine equivalent dose of $16 \mathrm{mg} / \mathrm{kg}$ into CD-1 nude mice. Data are mean \pm standard deviation of $\mathrm{n}=3$ mice. Data at $4 \mathrm{~h}$ are from MIA $\mathrm{PaCa}-2$ tumor-bearing mice.

${ }^{*} \mathrm{p}<0.05 ; * * \mathrm{p}<0.01 ; * * * \mathrm{p}<0.001$ versus free drug.

CL: Cleavable; PEG: Poly(ethene glycol); pSL: pH-sensitive liposome.

Table 3. Plasma pharmacokinetic parameters of gemcitabine after a single intravenous administration of drug solution or liposome formulations at a dose of $1 \mathrm{mg} / \mathrm{kg}$ to Sprague-Dawley rats (unless otherwise stated). Pharmacokinetics of CL-PEG-pSL at a higher dose of $5 \mathrm{mg} / \mathrm{kg}(\mathrm{n}=4)$ was further determined. Data are mean \pm standard deviation, $n=6$ unless otherwise stated.

\begin{tabular}{|c|c|c|c|c|c|}
\hline Formulation & AUC $\mu$ M.h & $V_{d}(\mathrm{ml} / \mathbf{k g})$ & Clearance $(\mathrm{ml} / \mathrm{h} / \mathrm{kg})$ & $\mathrm{T}_{1 / 2}(\mathrm{~h})$ & MRT (h) \\
\hline Free drug & $15.9 \pm 1.8$ & $1084 \pm 62$ & $247 \pm 24$ & $3.1 \pm 0.4$ & $4.0 \pm 0.4$ \\
\hline PEG-pSL & $95.1 \pm 12.4$ & $366 \pm 86$ & $42.1 \pm 5.6$ & $5.8 \pm 0.6$ & $5.4 \pm 0.3$ \\
\hline CL-PEG-pSL & $125 \pm 6^{*}$ & $377 \pm 33$ & $30.8 \pm 1.6$ & $8.6 \pm 1.0$ & $5.5 \pm 0.4$ \\
\hline CL-PEG-pSL (5 mg/kg) & $643 \pm 21$ & $414 \pm 32$ & $29.6 \pm 0.9$ & $9.8 \pm 1.1$ & $7.9 \pm 0.4$ \\
\hline
\end{tabular}

All CL-PEG-pSL values are significantly different than free drug $(p<0.05$, one-way ANOVA)

*Significantly different than PEG-pSL $(p<0.05$, one-way ANOVA).

AUC: Area under the curve; CL: Cleavable; pSL: pH-sensitive liposome; MRT: Mean residence time; $T_{1 / 2}$ : Elimination half-life; $V_{d}$ : Volume of distribution. 
of gemcitabine resulted in a $T_{1 / 2}$ and mean residence time of 9.8 and $7.2 \mathrm{~h}$, respectively, and the AUC increased proportionally fivefold.

The tissue distribution of gemcitabine 4 and $12 \mathrm{~h}$ after dosing as liposomal or free formulations was determined in CD1 nude mice, with MIA PaCa-2 tumor-bearing mice used for the 4-h time point. Like in Sprague-Dawley rats, the plasma levels of gemcitabine in liposomal formulations were higher than for the free drug. The data indicate that $4 \mathrm{~h}$ after the intravenous injection of CL-PEG-pSL, the concentration of gemcitabine in tumors was 1.5 -fold higher than for PEG-pSL $(\mathrm{p}<0.05)$ and six-times higher than for mice receiving free drug $(\mathrm{p}<0.001$; Figure $8 \mathrm{~B})$. Drug distribution in tumor was not monitored after $12 \mathrm{~h}$ as gemcitabine is a prodrug which could be converted to its metabolites and our analytical method was not sensitive enough to analyze its metabolites. Furthermore, the drug concentrations in the heart following treatment with both liposomes was threefold lower than free drug treated $(\mathrm{p}<0.001)$ mice at $4 \mathrm{~h}$, but was similar at $12 \mathrm{~h}$ (Figure $8 \mathrm{~B} \& \mathrm{C}$ ). The level of gemcitabine in the major clearance organ, the liver, was significantly $(\mathrm{p}<0.05)$ lower for free drug-injected mice compared with PEG-pSL -and CL-PEG-pSL-treated groups, at 4 and $12 \mathrm{~h}$ postinjection, reflecting the faster plasma clearance rates of the free drug. Spleen and kidney levels were similar for all three formulations at both time points. Tumor was the only tissue that showed a significant difference in gemcitabine concentrations between CL-PEG-pSL and PEG-pSL.

\section{Discussion}

The pSL functionalized by fusogenic DOPE and CHEMS have attracted great interest due to their tumortargeted drug delivery abilities $[2,4,5,10]$. The addition of PEG-coating promotes the long circulation and tumor accumulation of pSL based on the enhanced permeation and retention effect, however, hinders their cell uptake and acid-sensitivity (i.e., the PEG dilemma). Therefore, it is challenging to achieve $\mathrm{pH}$ sensitivity and prolonged circulation simultaneously, which are both highly desirable for tumor-targeted drug delivery. Furthermore, to achieve tumor targeting, it is critically important to understand which regions in tumors have lower $\mathrm{pH}$ values than normal tissues. In this work, CL-PEG-pSL were designed to exploit $\mathrm{pH}_{\mathrm{en}}$ to achieve rapid delivery into the cytosol, and $\mathrm{pH}_{\text {ex }}$ to initiate the PEG detachment.

An acid cleavable PEG polymer (PEG $\mathrm{B}_{\mathrm{B}} \mathrm{Hz}$-DPPE) to be used for PEG coating of preformed DOPE/CHEMSbased $\mathrm{pSL}$ was synthesized and characterized in this study (Figure 1). The $\mathrm{PEG}_{\mathrm{B}}-\mathrm{Hz}-\mathrm{DPPE}$ polymer with a CMC of $25 \mu \mathrm{M}$ (similar to DSPE-PEG, $20 \mu \mathrm{M}$ [35]) readily self-aggregated above this concentration to form micelles at $\mathrm{pH}$ 7.4. (Figure $2 \mathrm{~A}$ ). The $\mathrm{PEG}_{\mathrm{B}}-\mathrm{Hz}-\mathrm{DPPE}$, which included an acid-labile hydrazide-hydrazone (-CO-NH-N = $\mathrm{CH}-$ ) linker, rapidly decomposed under acidic conditions to shed the PEG-coating as evident from a pH-dependent shift in their size (Figure 2B) and rapid $\mathrm{pH}$-responsive Nile Red release from the micelles within $2 \mathrm{~h}$ (Figure 2C), but remained stable at $\mathrm{pH} 7.4$ for at least $24 \mathrm{~h}$. In comparison, cleavable PEG polymers synthesized by Torchillin $e t a l$., derived from aromatic aldehydes [24], and those developed by Chen $e$ al al, either lacked sufficient pH sensitivity or were not stable at $\mathrm{pH} 7.4$ [25]. On the other hand, modification with cleavable PEG polymers, PEG-CHMC and PEG-CHEMS, compromised the circulation half-life of liposomes due to cleavage of the PEG chain in blood [36]. The $\mathrm{PEG}_{\mathrm{B}}-\mathrm{Hz}-\mathrm{DPPE}$ synthesized in this study demonstrated $\mathrm{pH}$ responsiveness without compromising its stability at physiological $\mathrm{pH}$ 7.4. The hydrazone is reasonably stable at physiological $\mathrm{pH}$ due to the low basicity of its imino $\mathrm{N}$, therefore, preventing the rapid protonation of the hydrazide-hydrazone bond.

The PEG $\mathrm{B}_{\mathrm{B}}-\mathrm{Hz}$-DPPE polymer was further used to modify DOPE-CHEMS liposomes to form CL-PEG-pSL (Figure 3A), with a $5 \mathrm{~mol} \%$ PEGylation degree, which is optimal for stealth properties [37]. It benefitted the drug-loaded CL-PEG-pSL with an average size of $141.0 \pm 0.4 \mathrm{~nm}$ for passive targeting via the EPR effect [22]. Both PEG-pSL and CL-PEG-pSL showed a slightly negative charge (Table 1), contributing to their stability in vitro. The moderate gemcitabine release of the CL-PEG-pSL and PEG-pSL at pH 7.4 implied the drugs in liposomes could efficiently reach to the tumor area even after $48 \mathrm{~h}$, where detectable levels of drug are still identified for both liposome groups (Figure 4). DSPC, a high-transition temperature lipid, was incorporated in the liposomes to strengthen the liposomal membrane, and appears to have improved the stability and prevented to some extent the premature drug leakage issue of gemcitabine-loaded liposomes that has been seen previously [20]. Gemcitabine was released more quickly from CL-PEG-pSL than PEG-pSL at a $\mathrm{pH}_{\mathrm{en}}, 5.0$ (Figure 4), which is likely due to the $\mathrm{pH}$-responsive cleavage of $\mathrm{PEG}_{\mathrm{B}}-\mathrm{Hz}-\mathrm{DPPE}$ as shown in Figure 2. These CL-PEG-pSL did not show enhanced release at mild acidic $\mathrm{pH}$ 6.5, which is favorable for intracellular drug delivery via endocytosis of liposomes, however, will not be useful to enhance cellular uptake through PEG-shedding at low $\mathrm{pH}_{\mathrm{ex}}$ in tumors $(\mathrm{pH} 6.5-7)$. Upon entering the cells, with the hydrolysis of $\mathrm{PEG}_{\mathrm{B}}-\mathrm{Hz}$-DPPE CL-PEG-pSL could benefit the interaction of the 
fusogenic DOPE bilayer with endosomal membrane more efficiently, leading to the higher endosomal escape than PEG-pSL (Figure 5), which reduced the degradation of cargos in lysosomes [38].

Previously three possible pathways for endosome escape of pSL have been proposed [39]: rupture of endosomal membranes; liposome destabilization causing diffusion of contents; and fusion of liposomes with endosome membranes; leading to cytoplasmic delivery [40]. In this study, confocal microscopy revealed improved cellular uptake while live cell imaging with the aid of LysoTracker Deep Red further confirmed the colocalization of liposomes with endo/lysosomes and endosome escape abilities. The cellular uptake of two types of liposomes did not show difference. CL-PEG-pSL was elucidated to have rapid cytoplasmic delivery abilities over the PEG-pSL. CLPEG-pSL in lower $\mathrm{pH}$ was probably dependent on fusion pathways following the detachment of PEG (Figure 6 \& 7 ). Quantifying colocalization of Rh-PE-labeled liposomes with Lysotracker (probing endo/lysosomes) using Pearson's correlation coefficients suggests a rapid accumulation of PEG-pSL in the endo/lysosomal lumens before diffusing out to cytoplasm. In comparison, PEG-pSL showed much slower accumulation in the endo/lysosomal regions. The invisible PEG-pSL was possible entrapped in early endosomes and the slowly transferred to endo/lysosomes.

With gemcitabine-loaded liposomes, CL-PEG-pSL showed slightly more cytotoxicity than PEG-pSL to MIA $\mathrm{PaCa}-2$ cells, most likely due to the more rapid endosomal escape of CL-PEG-pSL. Surprisingly, both liposomes showed less cytotoxicity than the free drug. As a small molecule, free gemcitabine could be quickly taken up into the cells via the significantly enhanced hCNT1 (a nucleoside influx transporter) expression in MIA PaCa-2 cells [41], although downregulation of these transporters is possible clinically in gemcitabine-resistant cases [42]. However, liposomal formulations may be advantageous in vivo with the ability to accumulate in tumors, which cannot be reflected in the $2 \mathrm{D}$ cell culture model. Besides, the enhanced tumor cell cytotoxicity of doxorubicin-loaded CL-PEG-pSL compared with PEG-pSL also illustrated that PEG B $_{\mathrm{B}} \mathrm{Hz}-\mathrm{DPPE}$ modification of liposomes could improve the internalization and intracellular drug release of liposomes into MIA PaCa-2 pancreatic and U-87 MG glioblastoma cells, respectively.

The pharmacokinetics study in rats depicted that the PEG $_{\mathrm{B}}-\mathrm{Hz}$-DPPE-based CL-PEG-pSL had similar longcirculation abilities to PEG-pSL (Figure 8A). An increase in the drug dose from 1 to $5 \mathrm{mg} / \mathrm{kg}$ resulted in a proportional increase in the plasma AUC values. These results indicated the reasonable stability of $\mathrm{PEG}_{\mathrm{B}}-\mathrm{Hz}-$ DPPE at physiological $\mathrm{pH}$ as shown in the in vitro study (Figure 2), in contrast to the previously reported polymers such as PEG-CHMC and PEG-CHEMS [26,36]. Overall the data from this study supported that the PEG-detachment occurred only at lower $\mathrm{pH}$ from the CL-PEG-pSL but did not compromise their stealth abilities during blood circulation.

In CD-1 nude mice, both the liposomes showed significantly lower gemcitabine distribution in spleen and heart at $4 \mathrm{~h}$ compared with the drug solution-treated animals (Figure 8B \& C), showing their potential to reduce the side effects to these organs, such as cardiotoxicity [43]. Furthermore, CL-PEG-pSL increased the accumulation of gemcitabine in tumor by six-times that of the free drug, and 1.5-times PEG-pSL at the $4 \mathrm{~h}$ time point (Figure 8B).

\section{Conclusion}

A new $\mathrm{pH}$-sensitive $\mathrm{PEG}_{\mathrm{B}}-\mathrm{Hz}-\mathrm{DPPE}$ was successfully synthesized and utilized for the preparation of the acidtriggered PEG-cleavable pSL. PEG $\mathrm{B}-\mathrm{Hz}-\mathrm{DPPE}$ demonstrated good stability at physiological $\mathrm{pH}$ and rapid PEG shedding at endosomal $\mathrm{pH}$. The present study provides initial evidence that CL-PEG-pSL may be able to deliver drugs to tumors with higher efficiency than conventional PEG-pSL. Furthermore, this is the first study to fully elucidate the endosome escape kinetics of dual $\mathrm{pH}$-responsive liposomes facilitated with PEG-detachment using living cell imaging technique with the aid of colocalization analysis. This, in combination with the use of $\mathrm{pH}$ sensitive fusogenic DOPE which after dePEGylation in the endosomal lumen, facilitates the rapid endosome escape of the contents that are hindered by conventional PEGylation. These dual $\mathrm{pH}$-sensitive CL-PEG-pSL are promising in the application of tumor-targeted drug delivery in the future.

\section{Future perspective}

The low $\mathrm{pH}$-triggered de-PEGylation by coating liposomes with the $\mathrm{PEG}_{\mathrm{B}}-\mathrm{Hz}-\mathrm{DPPE}$ is demonstrated in this paper as a useful strategy to overcome the 'PEG dilemma' of $\mathrm{pSL}$, achieving more efficient intracellular drug release without compromising the long circulation property of liposomes. There are several issues that need to be addressed such as the complicated materials synthesis, the high cost and problematic stability during storage, which limits the application of liposomes. Furthermore, antitumor studies will be needed to critically evaluate the therapeutic utility of the dual $\mathrm{pH}$-sensitive liposomal system. Notably, the field of nanomedicines is currently challenged with 
some 'pitfalls' in their successful clinical translation [44,45], including the use of correct tumor models and further improvement of formulation design. This study addressed only one of the many issues. Significant research efforts are needed to bring new ideas for development of more effective nanomedicines for cancer treatment improvement.

\section{Summary points}

- Poly(ethene glycol)ylated pH-sensitive liposomes (PEG-pSL) have been widely investigated for efficient, tumor-targeted drug and gene delivery.

- PEGylation is essential to promote long circulation of pSL in vivo, however, reduces interaction with target cells and limits their endosomal escape property, termed as PEG dilemma.

- In this study, $\mathrm{PEG}_{\mathrm{B}}$-hydrazone-1,2-dipalmitoyl-sn-glycero-3-phosphoethanolamine conjugate was designed via an acid-labile hydrazide-hydrazone (-CO-NH-N = $\mathrm{CH}-$ ) bond and used for preparation of cleavable(CL)-PEG-pSL, (pSL with $\mathrm{pH}$-responsive sheddable PEG) to overcome this PEG dilemma.

- The CL-PEG-pSL was able to be achieve higher endosome escape abilities in MIA PaCa-2 cells (threefold) than the conventionally PEGylated CL-PEG-pSL (PEG-pSL).

- This CL-PEG-pSL takes advantage of both the acid-labile sheddable PEG layer and the pH-sensitive lipid bilayer that promotes the rapid intracellular trafficking, without compromising long circulation abilities.

- CL-PEG-pSL was able to be achieve a 1.5-fold higher tumor accumulation than the PEG-pSL.

- This is the first study to fully elucidate the endosome escape kinetics of pSL facilitated with low pH-triggered PEG-detachment using living cell imaging techniques with the aid of colocalization analysis.

- Dual fluorescent-labeling in the liposomes with 1,2-dipalmitoyl-sn-glycero-3-phosphoethanolamine-N-(lissamine rhodamine B sulfonyl) in membrane and calcein $(80 \mathrm{mM})$ in the cores allowed visualization of endosomal escape of liposomes, taking advantage of the self-quench property of calcein.

- Quantifying colocalization of liposome and endo/lysosomal lumen using Pearson's correlation coefficients is superior approach to illustrate endosomal-escape properties of pSL.

- While experimentally promising, many issues such as complicated materials synthesis, high cost and low stability need to be addressed for their successful translation from bench to bedside.

\section{Acknowledgments}

We wish to thank our funding source for this research, the Marsden Fund by the Royal Society of New Zealand (grant no.: UOA1201), the 2016 Performance Based Research Fund from our School, and a Faculty Research Development Fund (3716807) from University of Auckland. We would also like to thank Jacqueline M Ross and Hilary Holloway from the Biomedical Imaging Research Unit, University of Auckland for their technical support with the live cell and confocal imaging studies.

Financial \& competing interests disclosure

The authors thank our funding source for this research, the Marsden Fund by the Royal Society of New Zealand (grant no. UOA1201) and the 2016 Performance-Based Research Fund from School of Pharmacy and a Faculty Research Development Fund (3716807) from University of Auckland. The authors have no other relevant affiliations or financial involvement with any organization or entity with a financial interest in or financial conflict with the subject matter or materials discussed in the manuscript apart from those disclosed.

No writing assistance was utilized in the production of this manuscript.

\section{Ethical conduct of research}

Pharmacokinetic and biodistribution studies were carried out under protocols approved by the Animal Ethics Committee of the University of Auckland (ethics approval no. 001593 and 001880, respectively). The authors state that they have obtained appropriate institutional review board approval or have followed the principles outlined in the Declaration of Helsinki for all human or animal experimental investigations.

\section{Open access}

This work is licensed under the Attribution-NonCommercial-NoDerivatives 4.0 Unported License. To view a copy of this license, visit http://creativecommons.org/licenses/by-nc-nd/4.0/ 


\section{References}

Papers of special note have been highlighted as: $\bullet \bullet$ of considerable interest

1. Drummond DC, Zignani M, Leroux J. Current status of pH-sensitive liposomes in drug delivery. Prog. Lipid Res. 39(5), 409-460 (2000).

-. Outlines the current status of $\mathrm{pH}$-sensitive liposomes and their limitations in achieving tumor-targeted drug delivery.

2. Karanth H, Murthy RS. pH-sensitive liposomes-principle and application in cancer therapy. J. Pharm. Pharmacol. 59(4), 469-483 (2007)

3. Paliwal SR, Paliwal R, Vyas SP. A review of mechanistic insight and application of pH-sensitive liposomes in drug delivery. Drug Deliv. 22(3), 231-242 (2015).

4. Torchilin VP, Zhou F, Huang L. pH-sensitive liposomes. J. Liposome Res. 3(2), 201-255 (1993).

5. Yatvin MB, Kreutz W, Horwitz BA, Shinitzky M. pH-sensitive liposomes: possible clinical implications. Science 210(4475), 1253-1255 (1980).

6. Chu CJ, Dijkstra J, Lai MZ, Hong K, Szoka FC. Efficiency of cytoplasmic delivery by pH-sensitive liposomes to cells in culture. Pharm. Res. 7(8), 824-834 (1990).

7. Schroit AJ, Madsen J, Nayar R. Liposome-cell interactions: in vitro discrimination of uptake mechanism and in vivo targeting strategies to mononuclear phagocytes. Chem. Phys. Lipids 40(2-4), 373-393 (1986).

8. Liu X, Huang G. Formation strategies, mechanism of intracellular delivery and potential clinical applications of pH-sensitive liposomes. Asian J. Pharm. Sci. 8(6), 319-328 (2013).

9. Simões S, Slepushkin V, Düzgünes N, Pedroso De Lima MC. On the mechanisms of internalization and intracellular delivery mediated by pH-sensitive liposomes. Biochim. Biophys. Acta Biomembr. 1515(1), 23-37 (2001).

10. Vanic Z, Barnert S, Suss R, Schubert R. Fusogenic activity of pegylated pH-sensitive liposomes. J. Liposome Res. 22(2), 148-157 (2012).

11. Cho SM, Lee HY, Kim J-C. pH-dependent release property of dioleoylphosphatidyl ethanolamine liposomes. Korean J. Chem. Eng. 25(2), 390-393 (2008).

12. Geisow MJ, Evans WH. pH in the endosome: measurements during pinocytosis and receptor-mediated endocytosis. Exp. Cell Res. 150(1), 36-46 (1984).

13. Slepushkin VA, Simoes S, Dazin P et al. Sterically stabilized ph-sensitive liposomes. Intracellular delivery of aqueous contents and prolonged circulation in vivo. J. Biol. Chem. 272(4), 2382-2388 (1997).

14. Xu H, Paxton JW, Wu Z. Development of long-circulating $\mathrm{pH}$-sensitive liposomes to circumvent gemcitabine resistance in pancreatic cancer cells. Pharm. Res. 33(7), 1628-1637 (2016).

15. Kanamala M, Wilson WR, Yang M, Palmer BD, Wu Z. Mechanisms and biomaterials in $\mathrm{pH}$-responsive tumour targeted drug delivery: a review. Biomaterials 85, 152-167 (2016).

-• Outlines the design and development of various $\mathrm{pH}$-responsive tumor-targeted drug delivery mechanisms.

16. Remaut K, Lucas B, Braeckmans K, Demeester J, De Smedt SC. PEGylation of liposomes favours the endosomal degradation of the delivered phosphodiester oligonucleotides. J. Control. Rel. 117(2), 256-266 (2007).

17. Sadzuka Y, Kishi K, Hirota S, Sonobe T. Effect of polyethyleneglycol (PEG) chain on cell uptake of PEG-modified liposomes. J. Liposome Res. 13(2), 157-172 (2003).

18. Fang Y, Xue J, Gao S et al. Cleavable pegylation: a strategy for overcoming the "peg dilemma" in efficient drug delivery. Drug Deliv. 24(Suppl. 1), 22-32 (2017).

-• Pertains to the use of cleavable PEGylation as a strategy to overcome PEG dilemma.

19. Chan C-L, Majzoub RN, Shirazi RS et al. Endosomal escape and transfection efficiency of PEGylated cationic lipid-DNA complexes prepared with an acid-labile PEG-lipid. Biomaterials 33(19), 4928-4935 (2012).

20. Xu H, Paxton JW, Wu Z. Enhanced pH-responsiveness, cellular trafficking, cytotoxicity and long-circulation of PEGylated liposomes with post-insertion technique using gemcitabine as a model drug. Pharm. Res. 32(7), 2428-2438 (2015).

21. Kale AA, Torchilin VP. "Smart" drug carriers: PEGylated tatp-modified pH-sensitive liposomes. J. Liposome Res. 17(3-4), 197-203 (2007).

22. Bae YH, Park K. Targeted drug delivery to tumors: myths, reality and possibility. J. Control. Rel. 153(3), 198-205 (2011).

-• Outlines the various challenges to achieve targeted delivery to tumors.

23. Kim H-K, Thompson DH, Jang HS, Chung YJ, Van Den Bossche J. pH-responsive biodegradable assemblies containing tunable phenyl-substituted vinyl ethers for use as efficient gene delivery vehicles. ACS Appl. Mater. Interfaces 5(12), 5648-5658 (2013).

24. Kale AA, Torchilin VP. Design, synthesis, and characterization of ph-sensitive PEG-PE conjugates for stimuli-sensitive pharmaceutical nanocarriers: the effect of substitutes at the hydrazone linkage on the $\mathrm{pH}$ stability of PEG-PE conjugates. Bioconjug. Chem. 18(2), 363-370 (2007). 
25. Chen D, Liu W, Shen Y et al. Effects of a novel $\mathrm{pH}$-sensitive liposome with cleavable esterase-catalyzed and $\mathrm{pH}$-responsive double smart mpeg lipid derivative on abc phenomenon. Int. J. Nanomedicine 6, 2053-2061 (2011).

26. Kanamala M, Palmer BD, Ghandehari H, Wilson WR, Wu Z. PEG-benzaldehyde-hydrazone-lipid based PEG-sheddable pH-sensitive liposomes: abilities for endosomal escape and long circulation. Pharm. Res. 35(8), 154 (2018).

27. Cui C, Yu P, Wu M et al. Reduction-sensitive micelles with sheddable PEG shells self-assembled from a y-shaped amphiphilic polymer for intracellular doxorubicine release. Colloids Surf. B: Biointerfaces 129, 137-145 (2015).

28. Huang X, Du F, Cheng J et al. Acid-sensitive polymeric micelles based on thermoresponsive block copolymers with pendent cyclic orthoester groups. Macromolecules 42(3), 783-790 (2009).

29. Liu G-Y, Chen C-J, Li D-D, Wang S-S, Ji J. Near-infrared light-sensitive micelles for enhanced intracellular drug delivery. J. Mater. Chem. 22(33), 16865-16871 (2012).

30. Xu H, Paxton JW, Wu Z. Development of long-circulating $\mathrm{pH}$-sensitive liposomes to circumvent gemcitabine resistance in pancreatic cancer cells. Pharm. Res. 33(7), 1628-1637 (2016).

31. Zhang W, Wang G, Falconer JR et al. Strategies to maximize liposomal drug loading for a poorly water-soluble anticancer drug. Pharm. Res. 32(4), 1451-1461 (2015).

32. Xu H, Paxton J, Lim J, Li Y, Wu Z. Development of a gradient high performance liquid chromatography assay for simultaneous analysis of hydrophilic gemcitabine and lipophilic curcumin using a central composite design and its application in liposome development. $J$. Pharm. Biomed. Anal. 98, 371-378 (2014).

33. Xu H, Paxton J, Lim J et al. Development of high-content gemcitabine PEGylated liposomes and their cytotoxicity on drug-resistant pancreatic tumour cells. Pharm. Res. 31(10), 2583-2592 (2014).

34. Kanamala M, Palmer BD, Wilson WR, Wu Z. Characterization of a smart pH-cleavable PEG polymer towards the development of dual pH-sensitive liposomes. Int. J. Pharm. 548(1), 288-296 (2018).

35. Vuković L, Khatib FA, Drake SP et al. Structure and dynamics of highly PEG-ylated sterically stabilized micelles in aqueous media. $J$. Am. Chem. Soc. 133(34), 13481-13488 (2011).

36. Nag OK, Awasthi V. Surface engineering of liposomes for stealth behavior. Pharmaceutics 5(4), 542-569 (2013).

37. Dos Santos N, Allen C, Doppen A-M et al. Influence of poly(ethylene glycol) grafting density and polymer length on liposomes: relating plasma circulation lifetimes to protein binding. Biochim. Biophys. Acta Biomembr. 1768(6), 1367-1377 (2007).

38. Bucci C, Stasi M. Endosome to lysosome transport. In: Encyclopedia of Cell Biology. Academic Press, Cambridge, MA, USA $408-417$ (2016).

39. Varkouhi AK, Scholte M, Storm G, Haisma HJ. Endosomal escape pathways for delivery of biologicals. J. Control. Rel. 151(3), 220-228 (2011).

-• Discusses different endosomal escape pathways for delivery of the biologicals.

40. Luzio JP, Kuwana T, Mullock BM. Signals for transport from endosomes to lysosomes. In: Molecular Mechanisms of Membrane Traffic. NATO ASI Series (Series H: Cell Biology). Springer, Berlin, Heidelberg, Germany (1993).

41. Ciccolini J, Serdjebi C, Peters GJ, Giovannetti E. Pharmacokinetics and pharmacogenetics of gemcitabine as a mainstay in adult and pediatric oncology: an eortc-pamm perspective. Cancer Chemother. Pharmacol. 78(1), 1-12 (2016).

42. Bhutia YD, Hung SW, Patel B, Lovin D, Govindarajan R. Cnt1 expression influences proliferation and chemosensitivity in drug-resistant pancreatic cancer cells. Cancer Res. 71(5), 1825-1835 (2011).

43. Khan MF, Gottesman S, Boyella R, Juneman E. Gemcitabine-induced cardiomyopathy: a case report and review of the literature. J. Med. Case Rep. 8, 220 (2014).

44. Lammers T, Kiessling F, Ashford M, Hennink W, Crommelin D, Storm G. Cancer nanomedicine: is targeting our target? Nat. Rev. Mater. 1(9), 16069 (2016).

45. Mitragotri S, Lammers T, Bae YH et al. Drug delivery research for the future: expanding the nano horizons and beyond. J. Control. Rel. 246, 183-184 (2017). 
